DOI 10.4171/JEMS/310

Domenico Fiorenza · Donatella Iacono $\cdot$ Elena Martinengo

\title{
Differential graded Lie algebras controlling infinitesimal deformations of coherent sheaves
}

Received November 19, 2009 and in revised form May 18, 2010

\begin{abstract}
We use the Thom-Whitney construction to show that infinitesimal deformations of a coherent sheaf $\mathcal{F}$ are controlled by the differential graded Lie algebra of global sections of an acyclic resolution of the sheaf $\mathcal{E} n d^{*}\left(\mathcal{E}^{*}\right)$, where $\mathcal{E}$ is any locally free resolution of $\mathcal{F}$. In particular, one recovers the well known fact that the tangent space to $\operatorname{Def}_{\mathcal{F}}$ is $\operatorname{Ext}^{1}(\mathcal{F}, \mathcal{F})$, and obstructions are contained in $\operatorname{Ext}^{2}(\mathcal{F}, \mathcal{F})$. The main tool is the identification of the deformation functor associated with the Thom-Whitney DGLA of a semicosimplicial DGLA $\mathfrak{g}^{\Delta}$, whose cohomology is concentrated in nonnegative degrees, with a noncommutative Čech cohomology-type functor $H_{\mathrm{sc}}^{1}\left(\exp \mathfrak{g}^{\Delta}\right)$.
\end{abstract}

Keywords. Differential graded Lie algebras, functors of Artin rings

\section{Introduction}

The classical approach to deformation theory, starting with Kodaira and Spencer's studies on deformations of complex manifolds, consists in deforming the objects locally and then gluing back together these local deformations. During the last thirty years, another approach to deformation problems has been developed. The philosophy underlying it, essentially due to Quillen, Deligne, Drinfeld and Kontsevich, is that, in characteristic zero, every deformation problem is controlled by a differential graded Lie algebra, via solutions of the Maurer-Cartan equation modulo gauge equivalence. The aim of this paper is to exhibit an explicit equivalence between the two approaches for the problem of infinitesimal deformations of coherent sheaves.

In the particular case of a locally free sheaf $\mathcal{E}$ of $\mathcal{O}_{X}$-modules on a complex manifold $X$, Kodaira-Spencer's description of deformations of $\mathcal{E}$ is given in terms of the Čech functor $H^{1}(X$; exp $\mathcal{E} n d(\mathcal{E}))$, where $\mathcal{E} n d(\mathcal{E})$ is the sheaf of endomorphisms of $\mathcal{E}$. Indeed, a locally free sheaf has only trivial local deformations and so a deformation of $\mathcal{E}$ is reduced to a deformation of the gluing data of its local charts, and the compatibility condition

D. Fiorenza, E. Martinengo: Dipartimento di Matematica "Guido Castelnuovo",

Sapienza Università di Roma, P.le Aldo Moro 5, I-00185 Roma, Italy;

e-mail: fiorenza@mat.uniroma1.it, martinengo@mat.uniroma1.it

D. Iacono: Max-Planck Institut für Mathematik, Vivatsgasse 7, D-53111 Bonn, Germany;

e-mail: iacono@mpim-bonn.mpg.de

Mathematics Subject Classification (2010): Primary 13D10; Secondary 17B70, 18G30, 18G50, $18 \mathrm{G} 55$ 
these gluing data have to satisfy is precisely expressed by the cocycle condition in the Cech functor. On the other hand, it is well known that deformations of $\mathcal{E}$ are controlled by the DGLA of global sections of an acyclic resolution of $\mathcal{E}$ nd $(\mathcal{E})$, e.g., by the DGLA $A_{X}^{0, *}(\mathcal{E} n d(\mathcal{E}))$ of $(0, *)$-forms on $X$ with values in the sheaf of endomorphisms of $\mathcal{E}$.

The equivalence between these two descriptions is best understood by moving from set-valued to groupoid-valued deformation functors; see, e.g., [9, 20]. Associating with any open set $U$ in $X$ the groupoid $\operatorname{Def}_{\mathcal{E}_{\mid}}$of infinitesimal deformations of $\mathcal{E}$ over $U$ (over a fixed base Spec $A$, for some local Artin ring $A$ ) defines a stack over Top T $_{X}$; this is just a one-word way of saying that global deformations of $\mathcal{E}$ are the same thing as the descent data for its local deformations:

$$
\operatorname{Def}_{\mathcal{E}} \simeq \operatorname{holim}_{U \in \Delta_{\mathcal{U}}} \operatorname{Def}_{\left.\mathcal{E}\right|_{U}}
$$

where $\Delta_{\mathcal{U}}$ is the semisimplicial object in $\mathbf{T o p}_{X}$ associated with an open cover $\mathcal{U}$ of $X$. Next, one sees that locally the groupoid of deformations of $\left.\mathcal{E}\right|_{U}$ is equivalent to the Deligne groupoid of $\mathcal{E} n d(\mathcal{E})(U)$; since these equivalences are compatible with restriction maps, one has an equivalence of semicosimplicial groupoids. Finally, the Deligne groupoid commutes with homotopy limits of DGLA concentrated in nonnegative degree (see [9]), so that

$$
\operatorname{Def}_{\mathcal{E}} \simeq \operatorname{holim}_{U \in \Delta \mathcal{U}} \operatorname{Del}_{\mathcal{E} n d(\mathcal{E})(U)} \simeq \operatorname{Del}_{\operatorname{holim}} \mathcal{E} \operatorname{End}(\mathcal{E})(U)
$$

This shows that the problem of infinitesimal deformations of $\mathcal{E}$ is controlled by the DGLA $\operatorname{holim}_{U \in \Delta \mathcal{U}} \mathcal{E} n d(\mathcal{E})(U)$. It is now a simple exercise in homological algebra to show that there is a quasi-isomorphism of DGLAs

$$
\operatorname{holim}_{U \in \Delta_{\mathcal{U}}} \mathcal{E} n d(\mathcal{E})(U) \simeq A_{X}^{0, *}(\mathcal{E} n d(\mathcal{E}))
$$

The reader who prefers not to leave the peaceful realm of set-valued deformation functors can find a direct (but less enlightening) proof of the equivalence between KodairaSpencer's and the DGLA approach to infinitesimal deformation of locally free sheaves in the first arXiv version of [7].

We now turn our attention to deformations of a coherent sheaf $\mathcal{F}$ of $\mathcal{O}_{X}$-modules on a complex manifold or an algebraic variety $X$. The classical approach to this deformation problem is based on a locally free resolution $\mathcal{E} \rightarrow \mathcal{F}$ of $\mathcal{F}$; then, the data of a deformation of $\mathcal{F}$ are the data of local deformations of $\mathcal{E}$ with appropriate gluing conditions. More precisely, the sheaf of differential graded Lie algebras $\mathcal{E} n d^{*}(\mathcal{E})$ of the endomorphisms of the resolution $\mathcal{E}$ controls infinitesimal deformations of $\mathcal{F}$ via the Čech-type functor $H_{\mathrm{Ho}}^{1}\left(X ; \exp \mathcal{E} n d^{*}(\mathcal{E})\right)$; the subscript Ho refers to the fact that cocycle conditions hold only up to homotopy. The functor $H_{\mathrm{Ho}}^{1}\left(X ; \exp \mathcal{E} n d^{*}\left(\mathcal{E}^{*}\right)\right)$ is actually independent of the particular resolution chosen. And again, on the DGLA side, one proves that infinitesimal deformations of $\mathcal{F}$ are controlled by the DGLA of global sections of an acyclic resolution of $\mathcal{E} n d^{*}(\mathcal{E})$; in particular, one recovers the well known fact that the tangent space to $\operatorname{Def}_{\mathcal{F}}$ is $\operatorname{Ext}^{1}(\mathcal{F}, \mathcal{F})$, and obstructions are contained in $\operatorname{Ext}^{2}(\mathcal{F}, \mathcal{F})$. 
To see why such a result should hold, one has to make a further step and go from groupoid-valued to $\infty$-groupoid-valued deformation functors, and to think the whole problem in terms of $\infty$-stacks $[10,16,24]$. Indeed, due to the presence of negative degree components in $\mathcal{E} n d^{*}\left(\mathcal{E}^{*}\right)$, the groupoids $\left.\operatorname{Def}_{\mathcal{F}}\right|_{U}$ are not equivalent to the Deligne groupoids $\operatorname{Del}_{\mathcal{E} n d^{*}(\mathcal{E})(U)}$ anymore; yet from the $\infty$-groupoid point of view it is natural to expect that the stack $\operatorname{Def}_{\mathcal{F}}$ is locally homotopy equivalent to the $\infty$-stack $\operatorname{MC}_{\bullet}\left(\mathcal{E} n d^{*}\left(\mathcal{E}^{*}\right)\right)$. Then one reasons as in the locally free sheaf case, using the fact that the Kan complexesvalued functor MC. commutes with homotopy limits of DGLAs whose cohomology is concentrated in nonnegative degree [8]:

$$
\operatorname{Def}_{\mathcal{F}} \simeq \operatorname{holim}_{U \in \Delta_{\mathcal{U}}} \operatorname{Def}_{\left.\mathcal{F}\right|_{U}} \simeq \operatorname{holim}_{U \in \Delta_{\mathcal{U}}} \operatorname{MC}_{\bullet}\left(\mathcal{E} n d^{*}\left(\mathcal{E}^{*}\right)(U)\right) \simeq \operatorname{MC}_{\bullet}\left(\operatorname{holim}_{U \in \Delta_{\mathcal{U}}} \mathcal{E} n d^{*}(\mathcal{E})(U)\right)
$$

As above, the homotopy $\operatorname{limit}_{\text {holim }} \in \in \Delta_{\mathcal{U}} \mathcal{E} n d^{*}(\mathcal{E} \cdot)(U)$ is quasi-isomorphic to the DGLA of global sections of an acyclic resolution of $\mathcal{E} n d^{*}(\mathcal{E})$, which therefore controls the infinitesimal deformations of $\mathcal{F}$.

The aim of this paper is to give a direct proof of this fact at the level of set-valued deformation functors. The proof closely follows the original argument in [7] and does not rely on the conjectural homotopy equivalence between $\operatorname{Def}_{\left.\mathcal{F}\right|_{U}}$ and $\operatorname{MC}_{\bullet}\left(\mathcal{E}_{n} d^{*}(\mathcal{E})(U)\right)$. More precisely, we associate with any semicosimplicial DGLA $\mathfrak{g}^{\Delta}$ a set-valued func-

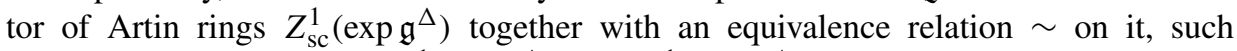
that the quotient functor $H_{\mathrm{sc}}^{1}\left(\exp \mathfrak{g}^{\Delta}\right)=Z_{\mathrm{sc}}^{1}\left(\exp \mathfrak{g}^{\Delta}\right) / \sim$ is an abstract version of $H_{\mathrm{Ho}}^{1}\left(X ; \exp \mathcal{E} d^{*}\left(\mathcal{E}^{*}\right)\right)$. The latter is obtained, as a particular case, by considering the Čech semicosimplicial Lie algebra $\mathcal{E} n d^{*}(\mathcal{E})(\mathcal{U})$ :

$$
\prod_{i} \mathcal{E} n d^{*}\left(\mathcal{E}^{\cdot}\right)\left(U_{i}\right) \Longrightarrow \prod_{i<j} \mathcal{E} n d^{*}\left(\mathcal{E}^{*}\right)\left(U_{i j}\right) \Longrightarrow \prod_{i<j<k} \mathcal{E} n d^{*}\left(\mathcal{E}^{*}\right)\left(U_{i j k}\right) \Longrightarrow
$$

Namely,

$$
H_{\mathrm{Ho}}^{1}\left(X ; \exp \mathcal{E} n d^{*}(\mathcal{E})\right)=\underset{\mathcal{U}}{\lim _{\mathrm{sc}}} H_{\mathrm{sc}}^{1}\left(\exp \mathcal{E} n d^{*}(\mathcal{E} \cdot)(\mathcal{U})\right)
$$

and both sides coincide with $H_{\mathrm{sc}}^{1}\left(\exp \mathcal{E} n d^{*}\left(\mathcal{E}^{\cdot}\right)(\mathcal{U})\right)$, for an $\mathcal{E} n d^{*}\left(\mathcal{E}^{\cdot}\right)$-acyclic cover of $X$. Next, we consider the Thom-Whitney model $\operatorname{Tot}_{T W} \mathfrak{g}^{\Delta}$ for holim $\mathfrak{g}^{\Delta}$ and show that there exists a commutative diagram of functors

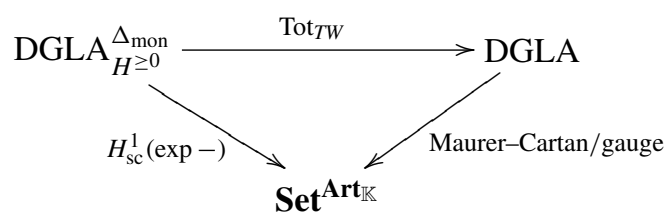

where DGLA $\Delta_{H \geq 0}^{\Delta_{\text {mon }}}$ is the category of semicosimplicial DGLAs with no negative cohomology. From the point of view of $\infty$-groupoids, this can be seen as an explicit description of the set $\pi_{\leq 0}\left(\mathrm{MC}_{\bullet}\left(\right.\right.$ holim $\left.\mathfrak{g}^{\Delta}\right)$ ).

We assume the reader is familiar with the language of deformation functors associated with DGLAs; see [17] for an introduction. Throughout this paper we work on a fixed 
algebraically closed field $\mathbb{K}$ of characteristic zero; the symbol Art $\mathbf{A K}_{\mathbb{K}}$ denotes the category of local Artinian $\mathbb{K}$-algebras $\left(A, \mathfrak{m}_{A}\right)$, with residue field $\mathbb{K}$. For a DGLA $L$, we denote by $\operatorname{Def}_{L}: \mathbf{A r t}_{\mathbb{K}} \rightarrow$ Set the associated deformation functor.

Most of the proofs in the present paper are completely straightforward, so we only sketch them; a preliminary version of this paper with fully detailed proofs is available on arXiv.

While revising this paper, we became aware of [25] where a similar construction is developed and investigated.

\section{Infinitesimal deformations and sheaves of DGLAs}

In this section, we study infinitesimal deformations of a coherent sheaf $\mathcal{F}$ of $\mathcal{O}_{X}$-modules on a smooth projective variety $X$ and explain how these deformations can be naturally described in terms of a sheaf of differential graded Lie algebras on $X$.

An infinitesimal deformation of the coherent sheaf of $\mathcal{O}_{X}$-modules $\mathcal{F}$ over $A \in \mathbf{A r t}_{\mathbb{K}}$ is given by a coherent sheaf $\mathcal{F}_{A}$ of $\mathcal{O}_{X} \otimes A$-modules on $X \times \operatorname{Spec} A$, flat over $A$, with a morphism of sheaves $\pi: \mathcal{F}_{A} \rightarrow \mathcal{F}$ inducing an isomorphism $\mathcal{F}_{A} \otimes_{A} \mathbb{K} \cong \mathcal{F}$. Two deformations $\mathcal{F}_{A}, \mathcal{F}_{A}^{\prime}$ of the coherent sheaf $\mathcal{F}$ over $A$ are isomorphic if there exists an isomorphism of sheaves $f: \mathcal{F}_{A} \rightarrow \mathcal{F}_{A}^{\prime}$, that commutes with the morphisms to $\mathcal{F}$. We denote by $\operatorname{Def}_{\mathcal{F}}: \mathbf{A r t}_{\mathbb{K}} \rightarrow$ Set the functor of infinitesimal deformations of the sheaf $\mathcal{F}$.

Since we are interested in flat deformations, one can investigate them by using a locally free resolution of $\mathcal{F}$ (see [1, par. 3], or [23, Theorem A.31] for details of these correspondences). Let

$$
0 \rightarrow \mathcal{E}^{-m} \stackrel{d}{\rightarrow} \cdots \stackrel{d}{\rightarrow} \mathcal{E}^{-1} \stackrel{d}{\rightarrow} \mathcal{E}^{0} \stackrel{d}{\rightarrow} \mathcal{F} \rightarrow 0
$$

be a global syzygy for $\mathcal{F}$, and denote by $\mathcal{E}$ the complex of locally free sheaves

$$
(\mathcal{E}, d): \quad 0 \rightarrow \mathcal{E}^{-m} \stackrel{d}{\rightarrow} \cdots \stackrel{d}{\rightarrow} \mathcal{E}^{-1} \stackrel{d}{\rightarrow} \mathcal{E}^{0} \rightarrow 0 .
$$

Let $\mathcal{U}=\left\{U_{i}\right\}_{i \in I}$ be an affine (or Stein, if we work in the complex analytic category) open cover of $X$ such that every sheaf of $\mathcal{E}$ is free on each $U_{i}$. The Kodaira-Spencer approach to infinitesimal deformations of $\mathcal{F}$ consists in deforming the sheaf $\mathcal{F}$ locally in such a way that local deformations glue together to a global sheaf, or equivalently, in view of the above discussion of the affine case, in deforming the complex $(\mathcal{E}, d)$ on every open set $U_{i}$ in such a way that these data glue together in cohomology. Following this approach, let us make explicit the deformation data: the first datum is an element $l=$ $\left\{l_{i}\right\}_{i} \in \prod_{i} \mathcal{E} n d^{1}(\mathcal{E})\left(U_{i}\right) \otimes \mathfrak{m}_{A}$ defining, on every open set $U_{i}$, a complex $\left(\left.\mathcal{E} \cdot\right|_{U_{i}} \otimes A, d+l_{i}\right)$ which is a deformation of the complex $\left(\left.\mathcal{E}^{\cdot}\right|_{U_{i}}, d\right)$. Note that the condition for $\left(\left.\mathcal{E}^{\cdot}\right|_{U_{i}} \otimes A\right.$, $\left.d+l_{i}\right)$ to be a complex is the Maurer-Cartan equation:

$$
d l_{i}+\frac{1}{2}\left[l_{i}, l_{i}\right]=0 \quad \text { for all } i \in I .
$$

Also note that, by upper semicontinuity of cohomology, the complex $\left(\left.\mathcal{E}^{\cdot}\right|_{U_{i}} \otimes A, d+l_{i}\right)$ is exact except possibly at zero level. To glue together the deformed local complexes 
$\left(\left.\mathcal{E}\right|_{U_{i}} \otimes A, d+l_{i}\right)$, we need to specify isomorphisms between the deformed complexes on the double intersections of open sets of the cover $\mathcal{U}$. Since these isomorphisms will have to be deformations of the identity, they will be of the form

$$
e^{m_{i j}}:\left(\left.\mathcal{E}\right|_{U_{i j}} \otimes A, d+l_{j}\right) \rightarrow\left(\left.\mathcal{E}\right|_{U_{i j}} \otimes A, d+l_{i}\right),
$$

with $m=\left\{m_{i j}\right\}_{i<j} \in \prod_{i<j} \mathcal{E} n d^{0}(\mathcal{E} \cdot)\left(U_{i j}\right) \otimes \mathfrak{m}_{A}$. The compatibiliy with the differentials, i.e., the commutativity of the diagrams

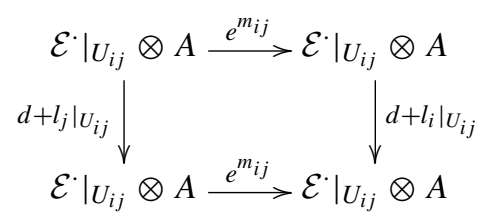

can be written as $d+\left.l_{i}\right|_{U_{i j}}=e^{m_{i j}}\left(d+\left.l_{j}\right|_{U_{i j}}\right) e^{-m_{i j}}$, i.e., as

$$
\left.l_{i}\right|_{U_{i j}}=\left.e^{m_{i j}} * l_{j}\right|_{U_{i j}} \quad \text { for all } i<j .
$$

Finally, the above isomorphisms have to satisfy the cocycle condition up to homotopy. Indeed, in order to obtain a deformation of $\mathcal{F}$, we actually do not want to glue together the complexes $\left(\left.\mathcal{E} \cdot\right|_{U_{i}} \otimes A, d+l_{i}\right)$, but rather their cohomology sheaves. In other words, we require $e^{m_{j k}} e^{-m_{i k}} e^{m_{i j}}$ to be homotopic to the identity on triple intersections. Taking logarithms, what we require is that $m_{j k} \bullet-m_{i k} \bullet m_{i j}$ is homotopy equivalent to zero, i.e.,

$$
\left.m_{j k}\right|_{U_{i j k}} \bullet-\left.\left.m_{i k}\right|_{U_{i j k}} \bullet m_{i j}\right|_{U_{i j k}}=\left[d+\left.l_{j}\right|_{U_{i j k}}, n_{i j k}\right]
$$

for some $n=\left\{n_{i j k}\right\}_{i j k} \in \prod_{i<j<k} \mathcal{E} n d^{-1}(\mathcal{E} \cdot)\left(U_{i j k}\right)$. This homotopy cocycle equation is conveniently rewritten as

$$
\left.m_{j k}\right|_{U_{i j k}} \bullet-\left.\left.m_{i k}\right|_{U_{i j k}} \bullet m_{i j}\right|_{U_{i j k}}=d_{\mathcal{E} n d^{*}(\mathcal{E})} n_{i j k}+\left[\left.l_{j}\right|_{U_{i j k}}, n_{i j k}\right] .
$$

Next, let us explain how the data introduced above are concretely linked with deformations of the coherent sheaf $\mathcal{F}$ over $A$. As the homotopy cocycle equation is satisfied, the local $A$-flat sheaves of $\left.\mathcal{O}_{X}\right|_{U_{i}} \otimes A$-modules $\mathcal{F}_{A, U_{i}}:=\mathcal{H}^{*}\left(\left.\mathcal{E}^{\cdot}\right|_{U_{i}} \otimes A, d+l_{i}\right)$ glue together to give a global coherent sheaf $\mathcal{F}_{A}$ which is a deformation of $\mathcal{F}$. On the other hand, every deformation $\mathcal{F}_{A}$ of the sheaf $\mathcal{F}$ can be obtained in this way. Indeed, the resolution $(\mathcal{E}, d)$ locally extends to projective resolutions $\left(\left.\mathcal{E}\right|_{U_{i}} \otimes A, d+l_{i}\right)$ of $\left.\mathcal{F}_{A}\right|_{U_{i}}$; these deformed local resolutions are linked to each other on double intersections by isomorphisms of complexes lifting the identity of $\mathcal{F}_{A}$, and the compositions of these isomorphisms on triple intersections are homotopic to the identity, since they lift the identity of $\mathcal{F}_{A}$ and the liftings are unique up to homotopy.

Let now $\mathcal{F}_{A}$ and $\mathcal{F}_{A}^{\prime}$ be isomorphic deformations of the sheaf $\mathcal{F}$, associated with deformation data $(l, m)$ and $\left(l^{\prime}, m^{\prime}\right)$, respectively. The restriction to every open set $U_{i}$ of the isomorphism between $\mathcal{F}_{A}$ and $\mathcal{F}_{A}^{\prime}$ lifts to local isomorphisms between the corresponding deformed complexes. Since these isomorphisms specialize to identities of $\left(\left.\mathcal{E} \cdot\right|_{U_{i}}, d\right)$, they are of the form $e^{a_{i}}:\left(\left.\mathcal{E} \cdot\right|_{U_{i}} \otimes A, d+l_{i}\right) \rightarrow\left(\left.\mathcal{E} \cdot\right|_{U_{i}} \otimes A, d+l_{i}^{\prime}\right)$, where 
$a=\left\{a_{i}\right\}_{i} \in \prod_{i} \mathcal{E} n d^{0}(\mathcal{E})\left(U_{i}\right) \otimes \mathfrak{m}_{A}$. As above, compatibility with the differentials translates into the equations

$$
e^{a_{i}} * l_{i}=l_{i}^{\prime} \quad \text { for all } i \in I .
$$

Finally, since the local isomorphisms $e^{a_{i}}$ lift a global isomorphism in cohomology, the diagrams

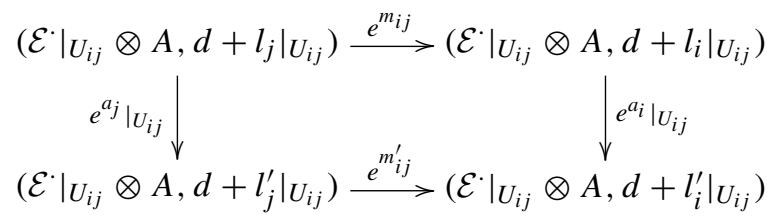

expressing compatibility with the gluing morphisms, commute in cohomology. Moreover, since the compositions $e^{-m_{i j}} e^{-a_{i}} e^{m_{i j}^{\prime}} e^{a_{j}}$ lift the identity of $\mathcal{F}_{A}$ on double intersections and the liftings are unique up to homotopy, these compositions are homotopic to the identity and, reasoning as above, we find

$$
-m_{i j} \bullet-\left.\left.a_{i}\right|_{U_{i j}} \bullet m_{i j}^{\prime} \bullet a_{j}\right|_{U_{i j}}=d_{\mathcal{E} n d^{*}(\mathcal{E})} b_{i j}+\left[\left.l_{j}\right|_{U_{i j}}, b_{i j}\right]
$$

for some $b=\left\{b_{i j}\right\}_{i<j} \in \prod_{i<j} \mathcal{E} n d^{-1}(\mathcal{E})\left(U_{i j}\right) \otimes \mathfrak{m}_{A}$. Conversely, if for the deformation data $(l, m)$ and $\left(l^{\prime}, m^{\prime}\right)$ there exist $a=\left\{a_{i}\right\}_{i} \in \prod_{i} \mathcal{E} n d^{0}(\mathcal{E})\left(U_{i}\right) \otimes \mathfrak{m}_{A}$ and $b=\left\{b_{i j}\right\}_{i<j} \in$ $\prod_{i<j} \mathcal{E} n d^{-1}(\mathcal{E})\left(U_{i j}\right) \otimes \mathfrak{m}_{A}$ that satisfy the equations above, the local isomorphisms $e^{a_{i}}$ glue together in cohomology to give a global isomorphism of the corresponding deformed sheaves $\mathcal{F}_{A}$ and $\mathcal{F}_{A}^{\prime}$.

Summing up, we have shown that in the Kodaira-Spencer approach, infinitesimal deformations of the coherent sheaf $\mathcal{F}$ are controlled by the sheaf of DGLAs $\mathcal{E} n d^{*}(\mathcal{E})$, via the equations above. At the end of Section 4.3, we will apply techniques of semicosimplicial DGLAs developed in this paper to recover the classical well known fact that the functor of infinitesimal deformations of $\mathcal{F}$ has $\operatorname{Ext}^{1}(\mathcal{F}, \mathcal{F})$ as tangent space and its obstructions are contained in $\operatorname{Ext}^{2}(\mathcal{F}, \mathcal{F})$.

Remark 2.1. The above description of the functor of infinitesimal deformations of $\mathcal{F}$ is actually independent of the resolution chosen. Indeed, the DGLAs of the endomorphisms of any two locally free resolutions of $\mathcal{F}$ are quasi-isomorphic (see,e.g., [22, Lemma 4.4]).

Remark 2.2. If the sheaf $\mathcal{F}$ is locally free, then we can take its trivial resolution $0 \rightarrow$ $\mathcal{F} \rightarrow \mathcal{F} \rightarrow 0$; thus, we recover the well known fact that the infinitesimal deformations of $\mathcal{F}$ are controlled by the sheaf $\mathcal{E} n d(\mathcal{F})$, via the Čech functor $H^{1}(X, \mathcal{E} n d(\mathcal{F}))$.

Remark 2.3. Note that the results of this section hold under the hypothesis that $\mathcal{F}$ admits a resolution $\mathcal{E} \rightarrow \mathcal{F}$ by locally free sheaves of $\mathcal{O}_{X}$-modules. This hypothesis is always satisfied, but in the general case the resolution is less obvious, it is not of finite length and the sheaves $\mathcal{E}^{-i}$ are not of finite rank; see, e.g., [12, Section 1.5]. 


\section{The Thom-Whitney DGLA}

\subsection{Semicosimplicial DGLAs and the functor $H_{\mathrm{sc}}^{1}\left(\exp \mathfrak{g}^{\Delta}\right)$}

A semicosimplicial differential graded Lie algebra is a covariant functor $\Delta_{\text {mon }} \rightarrow$ DGLA, from the category $\Delta_{\text {mon }}$, whose objects are finite ordinal sets and whose morphisms are order-preserving injective maps between them, to the category of DGLAs. Equivalently, a semicosimplicial DGLA $\mathfrak{g}^{\Delta}$ is a diagram

$$
\mathfrak{g}_{0} \Longrightarrow \mathfrak{g}_{1} \Longrightarrow \mathfrak{g}_{2} \equiv \ldots,
$$

where each $\mathfrak{g}_{i}$ is a DGLA, and for each $i>0$ there are $i+1$ morphisms of DGLAs

$$
\partial_{k, i}: \mathfrak{g}_{i-1} \rightarrow \mathfrak{g}_{i}, \quad k=0, \ldots, i,
$$

such that $\partial_{k+1, i+1} \partial_{l, i}=\partial_{l, i+1} \partial_{k, i}$ for any $k \geq l$.

Definition 3.1. Let $\mathfrak{g}^{\Delta}$ be a semicosimplicial DGLA. The functor

$$
Z_{\mathrm{sc}}^{1}\left(\exp \mathfrak{g}^{\Delta}\right): \mathbf{A r t}_{\mathbb{K}} \rightarrow \text { Set }
$$

is defined, for all $A \in \mathbf{A r t}_{\mathbb{K}}$, by

$$
\begin{aligned}
& Z_{\mathrm{sc}}^{1}\left(\exp ^{\Delta}\right)(A) \\
& =\left\{\begin{array}{l|l}
(l, m) \in\left(\mathfrak{g}_{0}^{1} \oplus \mathfrak{g}_{1}^{0}\right) \otimes \mathfrak{m}_{A} & \begin{array}{l}
d l+\frac{1}{2}[l, l]=0, \\
\partial_{1,1} l=e^{m} * \partial_{0,1} l, \\
\partial_{0,2} m \bullet-\partial_{1,2} m \bullet \partial_{2,2} m=d n+\left[\partial_{2,2} \partial_{0,1} l, n\right] \\
\text { for some } n \in \mathfrak{g}_{2}^{-1} \otimes \mathfrak{m}_{A}
\end{array}
\end{array}\right\} .
\end{aligned}
$$

Remark 3.2. In DGLA theory, given a DGLA $L$ and a Maurer-Cartan element $x$ in $\mathrm{MC}_{L}(A)$, the set

$$
\operatorname{Stab}(x)=\left\{d h+[x, h] \mid h \in L^{-1} \otimes \mathfrak{m}_{A}\right\}
$$

is called the irrelevant stabilizer of $x$. Note that $\operatorname{Stab}(x) \subseteq \operatorname{stab}(x)$, where $\operatorname{stab}(x)=$ $\left\{a \in L^{0} \otimes \mathfrak{m}_{A} \mid e^{a} * x=x\right\}$ is the stabilizer of $x$ under the gauge action of $L^{0} \otimes \mathfrak{m}_{A}$ on $\operatorname{MC}_{L}(A)$. Also note that, for any $a \in L^{0} \otimes \mathfrak{m}_{A}, e^{a} e^{\operatorname{Stab}(x)} e^{-a}=e^{\operatorname{Stab}(y)}$ with $y=e^{a} * x$.

We now introduce an equivalence relation $\sim$ on the set $Z_{\mathrm{sc}}^{1}\left(\exp \mathfrak{g}^{\Delta}\right)(A)$ as follows: we say that two elements $\left(l_{0}, m_{0}\right)$ and $\left(l_{1}, m_{1}\right)$ in $Z_{\mathrm{sc}}^{1}\left(\operatorname{exp~g}^{\Delta}\right)(A)$ are equivalent if and only if there exist $a \in \mathfrak{g}_{0}^{0} \otimes \mathfrak{m}_{A}$ and $b \in \mathfrak{g}_{1}^{-1} \otimes \mathfrak{m}_{A}$ such that

$$
\left\{\begin{array}{l}
e^{a} * l_{0}=l_{1}, \\
-m_{0} \bullet-\partial_{1,1} a \bullet m_{1} \bullet \partial_{0,1} a=d b+\left[\partial_{0,1} l_{0}, b\right] .
\end{array}\right.
$$

Definition 3.3. Let $\mathfrak{g}^{\Delta}$ be a semicosimplicial DGLA. The functor

$$
H_{\mathrm{sc}}^{1}\left(\exp \mathfrak{g}^{\Delta}\right): \mathbf{A r t}_{\mathbb{K}} \rightarrow \mathbf{S e t}
$$

is defined, for all $A \in \mathbf{A r t}_{\mathbb{K}}$, by $H_{\mathrm{sc}}^{1}\left(\exp \mathfrak{g}^{\Delta}\right)(A)=Z_{\mathrm{sc}}^{1}\left(\exp \mathfrak{g}^{\Delta}\right)(A) / \sim$. 
Remark 3.4. If $\mathfrak{g}^{\Delta}$ is a semicosimplicial Lie algebra, i.e., if all the DGLAs $\mathfrak{g}_{i}$ are concentrated in degree zero, then $H_{\mathrm{sc}}^{1}\left(\exp ^{\Delta}\right)$ reduces to the functor considered in [7].

Remark 3.5. The projection $\pi: Z_{\mathrm{sc}}^{1}\left(\exp ^{\Delta}\right) \rightarrow H_{\mathrm{sc}}^{1}\left(\exp ^{\Delta}\right)$ is a smooth morphism of functors of Artin rings, i.e., for any surjection $\beta: B \rightarrow A$ in $\mathbf{A r t}_{\mathbb{K}}$, the map

$$
Z_{\mathrm{sc}}^{1}\left(\exp \mathfrak{g}^{\Delta}\right)(B) \rightarrow H_{\mathrm{sc}}^{1}\left(\exp \mathfrak{g}^{\Delta}\right)(B) \times_{H_{\mathrm{sc}}^{1}\left(\exp \mathfrak{g}^{\Delta}\right)(A)} Z_{\mathrm{sc}}^{1}\left(\exp \mathfrak{g}^{\Delta}\right)(A)
$$

induced by

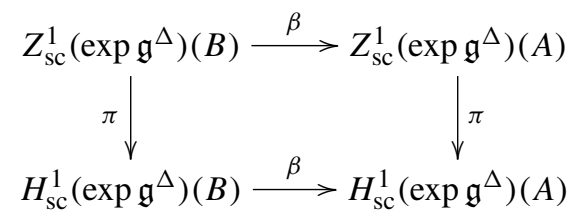

is a surjection of sets. Indeed, let $\left([(l, m)],\left(l_{0}, m_{0}\right)\right) \in H_{\mathrm{sc}}^{1}\left(\exp \mathfrak{g}^{\Delta}\right)(B) \times{ }_{H_{\mathrm{sc}}^{1}}\left(\exp \mathfrak{g}^{\Delta}\right)(A)$ $Z_{\mathrm{sc}}^{1}\left(\exp \mathfrak{g}^{\Delta}\right)(A)$. Then $(\beta l, \beta m)$ and $\left(l_{0}, m_{0}\right)$ are gauge equivalent in $Z_{\mathrm{sc}}^{1}\left(\exp \mathfrak{g}^{\Delta}\right)(A)$, i.e., there exists $a \in \mathfrak{g}_{0}^{0} \otimes \mathfrak{m}_{A}$ such that $e^{a} * \beta l=l_{0}$ and $-\beta m \bullet-\partial_{1,1} a \bullet m_{0} \bullet \partial_{0,1} a=$ $d b+\left[\partial_{0,1} \beta l, b\right]$ for some $b \in \mathfrak{g}_{1}^{-1} \otimes \mathfrak{m}_{A}$. Let $\tilde{a} \in \mathfrak{g}_{0}^{0} \otimes m_{B}$ and $\tilde{b} \in \mathfrak{g}_{1}^{-1} \otimes \mathfrak{m}_{B}$ be liftings of $a$ and $b$, respectively. The element $\left(e^{\tilde{a}} * l, \partial_{1,1} \tilde{a} \bullet m \bullet\left(d \tilde{b}+\left[\partial_{0,1} l, \tilde{b}\right]\right) \bullet-\partial_{0,1} \tilde{a}\right) \in$ $Z_{\mathrm{sc}}^{1}\left(\operatorname{exp~g}^{\Delta}\right)(B)$ is a pre-image of $\left([(l, m)],\left(l_{0}, m_{0}\right)\right)$.

The geometric application to have in mind is the following: given a sheaf $\mathcal{L}$ of DGLAs on a topological space $X$, and an open cover $\mathcal{U}$ of $X$, one has the Čech cosimplicial DGLA $\mathcal{L}(\mathcal{U})$,

$$
\prod_{i} \mathcal{L}\left(U_{i}\right) \Longrightarrow \prod_{i<j} \mathcal{L}\left(U_{i j}\right) \Longrightarrow \prod_{i<j<k} \mathcal{L}\left(U_{i j k}\right) \rightrightarrows \rightrightarrows \cdots,
$$

where the morphisms $\partial_{k, i}$ are the restriction maps. The functor $H_{\mathrm{sc}}^{1}(\exp \mathcal{L}(\mathcal{U}))$ depends on the cover $\mathcal{U}$, but the limit over open covers is a well defined functor:

$$
H_{\mathrm{Ho}}^{1}(X ; \exp \mathcal{L})=\lim _{\overrightarrow{\mathcal{U}}} H_{\mathrm{sc}}^{1}(\exp \mathcal{L}(\mathcal{U})): \operatorname{Art}_{\mathbb{K}} \rightarrow \text { Set. }
$$

Indeed, let $\mathcal{U}=\left\{U_{\alpha}\right\}_{\alpha \in I}$ and $\mathcal{U}^{\prime}=\left\{U_{\alpha}^{\prime}\right\}_{\alpha \in I^{\prime}}$ be open covers of $X$ with $\mathcal{U}^{\prime}$ a refinement of $\mathcal{U}$ and $\phi, \psi: I^{\prime} \rightarrow I$ two refinement maps. Both $\phi$ and $\psi$ induce, for all $A \in \mathbf{A r t}_{\mathbb{K}}$, a morphism $Z_{\mathrm{sc}}^{1}(\exp \mathcal{L}(\mathcal{U}))(A) \rightarrow Z_{\mathrm{sc}}^{1}\left(\exp \mathcal{L}\left(\mathcal{U}^{\prime}\right)\right)(A)$, defined by sending $\left(l_{i}, m_{i j}\right)$ to $\rho_{\phi}\left(l_{i}, m_{i j}\right)=\left(\left.l_{\phi \alpha}\right|_{U_{\alpha}^{\prime}},\left.m_{\phi \alpha, \phi \beta}\right|_{U_{\alpha \beta}^{\prime}}\right)$ and $\rho_{\psi}\left(l_{i}, m_{i j}\right)=\left(\left.l_{\psi \alpha}\right|_{U_{\alpha}^{\prime}},\left.m_{\psi \alpha, \psi \beta}\right|_{U_{\alpha \beta}^{\prime}}\right)$, respectively. Choosing $a_{\alpha}:=\left.m_{\psi \alpha, \phi \alpha}\right|_{U_{\alpha}^{\prime}} \in \mathcal{L}^{0}\left(U_{\alpha}^{\prime}\right) \otimes m_{A}$ for all $\alpha$ in $I^{\prime}$, we have

$$
\left\{\begin{array}{l}
\left.e^{a_{\alpha}} * l_{\phi \alpha}\right|_{U_{\alpha}^{\prime}}=\left.l_{\psi \alpha}\right|_{U_{\alpha}^{\prime}}, \\
-\left.m_{\phi \alpha, \phi \beta}\right|_{U_{\alpha \beta}^{\prime}} \bullet-\left.\left.\left.a_{\alpha}\right|_{U_{\alpha \beta}^{\prime}} \bullet m_{\psi \alpha, \psi \beta}\right|_{U_{\alpha \beta}^{\prime}} \bullet a_{\beta}\right|_{U_{\alpha \beta}^{\prime}} \in \operatorname{Stab}\left(\left.l_{\phi \beta}\right|_{U_{\alpha \beta}^{\prime}}\right) .
\end{array}\right.
$$

Therefore, $\rho_{\phi}\left(l_{i}, m_{i j}\right) \sim \rho_{\psi}\left(l_{i}, m_{i j}\right)$, for all $\left(l_{i}, m_{i j}\right) \in Z_{\mathrm{sc}}^{1}(\exp \mathcal{L}(\mathcal{U}))(A)$, and so the induced morphisms $\rho_{\phi}, \rho_{\psi}: H_{\mathrm{sc}}^{1}(\exp \mathcal{L}(\mathcal{U})) \rightarrow H_{\mathrm{sc}}^{1}\left(\exp \mathcal{L}\left(\mathcal{U}^{\prime}\right)\right)$ coincide. 
Remark 3.6. Having introduced the limit $H_{\mathrm{Ho}}^{1}(\exp \mathcal{L})$, for a sheaf of DGLAs $\mathcal{L}$ on a topological space $X$, the results of Section 2 can be restated as follows: the functor of infinitesimal deformations of a coherent sheaf $\mathcal{F}$ on a projective manifold $X$ is

$$
\operatorname{Def}_{\mathcal{F}} \cong H_{\mathrm{Ho}}^{1}\left(X ; \exp \mathcal{E} n d^{*}\left(\mathcal{E}^{\cdot}\right)\right)
$$

where $\mathcal{E}$ is a locally free resolution of $\mathcal{F}$.

The example of coherent sheaves on projective manifolds together with the DGLA approach to deformation theory suggests that the functors of Artin rings $H_{\mathrm{sc}}^{1}\left(\exp \mathfrak{g}^{\Delta}\right)$ could actually be isomorphic to functors $\operatorname{Def}_{L\left(\mathfrak{g}^{\Delta}\right)}$ for some DGLA $L\left(\mathfrak{g}^{\Delta}\right)$ canonically associated with $\mathfrak{g}^{\Delta}$. We are going to show that, under the cohomological hypothesis $H^{-1}\left(\mathfrak{g}_{2}\right)=0$, this is indeed so. More precisely, we are going to prove that, if $H^{-1}\left(\mathfrak{g}_{2}\right)=0$, then the functor of Artin rings $H_{\mathrm{sc}}^{1}\left(\operatorname{exp~g}^{\Delta}\right)$ is isomorphic to the deformation functor associated with the Thom-Whitney DGLA of the truncation $\mathfrak{g}^{\Delta_{[0,2]}}$.

\subsection{The Thom-Whitney DGLA $\operatorname{Tot}_{T W}\left(\mathfrak{g}^{\Delta}\right)$}

Let $\mathfrak{g}^{\Delta}$ be a semicosimplicial DGLA. The maps

$$
\partial_{i}=\partial_{0, i}-\partial_{1, i}+\cdots+(-1)^{i} \partial_{i, i}
$$

endow the vector space $\bigoplus_{i} \mathfrak{g}_{i}$ with the structure of a differential complex. Moreover, being a DGLA, each $\mathfrak{g}_{i}$ is in particular a differential complex

$$
\mathfrak{g}_{i}=\bigoplus_{j} \mathfrak{g}_{i}^{j}, \quad d_{i}: \mathfrak{g}_{i}^{j} \rightarrow \mathfrak{g}_{i}^{j+1},
$$

and since the maps $\partial_{k, i}$ are morphisms of DGLAs, the space $\mathfrak{g}_{\bullet}^{\bullet}=\bigoplus_{i, j} \mathfrak{g}_{i}^{j}$ has a natural bicomplex structure. The associated total complex

$$
\left(\operatorname{Tot}\left(\mathfrak{g}^{\Delta}\right), d_{\mathrm{Tot}}\right) \quad \text { where } \quad \operatorname{Tot}\left(\mathfrak{g}^{\Delta}\right)=\bigoplus_{i} \mathfrak{g}_{i}[-i], d_{\mathrm{Tot}}=\sum_{i, j} \partial_{i}+(-1)^{j} d_{j}
$$

has no natural DGLA structure. Yet there is another bicomplex naturally associated with a semicosimplicial DGLA, whose total complex is naturally a DGLA.

For every $n \geq 0$, denote by $\Omega_{n}$ the differential graded commutative algebra of polynomial differential forms on the standard $n$-simplex $\Delta^{n}$ :

$$
\Omega_{n}=\frac{\mathbb{K}\left[t_{0}, \ldots, t_{n}, d t_{0}, \ldots, d t_{n}\right]}{\left(\sum t_{i}-1, \sum d t_{i}\right)} .
$$

Denote by $\delta^{k, n}: \Omega_{n} \rightarrow \Omega_{n-1}, k=0, \ldots, n$, the face maps; then one has natural morphisms of bigraded DGLAs

$$
\delta^{k, n}: \Omega_{n} \otimes \mathfrak{g}_{n} \rightarrow \Omega_{n-1} \otimes \mathfrak{g}_{n}, \quad \partial_{k, n}: \Omega_{n-1} \otimes \mathfrak{g}_{n-1} \rightarrow \Omega_{n-1} \otimes \mathfrak{g}_{n},
$$

for every $0 \leq k \leq n$. 
The Thom-Whitney bicomplex is defined as

$$
C_{T W}^{i, j}\left(\mathfrak{g}^{\Delta}\right)=\left\{\left(x_{n}\right)_{n \in \mathbb{N}} \in \prod_{n} \Omega_{n}^{i} \otimes \mathfrak{g}_{n}^{j} \mid \delta^{k, n} x_{n}=\partial_{k, n} x_{n-1} \forall 0 \leq k \leq n\right\},
$$

where $\Omega_{n}^{i}$ denotes the degree $i$ component of $\Omega_{n}$. Its total complex is a DGLA, called the Thom-Whitney DGLA, and it is denoted by $\operatorname{Tot}_{T W}\left(\mathfrak{g}^{\Delta}\right)$; denote by $d_{T W}$ the differential of the Thom-Whitney DGLA. It is a remarkable fact that the integration maps

$$
\int_{\Delta^{n}} \otimes \mathrm{Id}: \Omega_{n} \otimes \mathfrak{g}_{n} \rightarrow \mathbb{K}[n] \otimes \mathfrak{g}_{n}=\mathfrak{g}_{n}[n]
$$

give a quasi-isomorphism of differential complexes $I: \operatorname{Tot}_{T W}\left(\mathfrak{g}^{\Delta}\right) \rightarrow \operatorname{Tot}\left(\mathfrak{g}^{\Delta}\right)$. In particular, the tangent space to $\operatorname{Def}_{\operatorname{Tot}_{T W}\left(\mathfrak{g}^{\Delta}\right)}$ is

$$
T \operatorname{Def}_{\operatorname{Tot}_{T W}\left(\mathfrak{g}^{\Delta}\right)} \cong H^{1}\left(\operatorname{Tot}_{T W}\left(\mathfrak{g}^{\Delta}\right)\right) \cong H^{1}\left(\operatorname{Tot}\left(\mathfrak{g}^{\Delta}\right)\right)
$$

and obstructions live in

$$
H^{2}\left(\operatorname{Tot}_{T W}\left(\mathfrak{g}^{\Delta}\right)\right) \cong H^{2}\left(\operatorname{Tot}\left(\mathfrak{g}^{\Delta}\right)\right) .
$$

Moreover, Dupont has described in [3, 4] an explicit morphism of differential complexes $E: \operatorname{Tot}\left(\mathfrak{g}^{\Delta}\right) \rightarrow \operatorname{Tot}_{T W}\left(\mathfrak{g}^{\Delta}\right)$ and an explicit homotopy $h: \operatorname{Tot}_{T W}\left(\mathfrak{g}^{\Delta}\right) \rightarrow \operatorname{Tot}_{T W}\left(\mathfrak{g}^{\Delta}\right)[-1]$ such that $I E=\operatorname{Id}_{\operatorname{Tot}\left(\mathfrak{g}^{\Delta}\right)}$ and $E I-\operatorname{Id}_{\operatorname{Tot}_{T W}\left(\mathfrak{g}^{\Delta}\right)}=\left[h, d_{T W}\right]$. We also refer to the papers [2, $8,19]$ for the explicit description of $E, h$ and for the proof of the above identities. Here, we point out that the construction of $\operatorname{Tot}_{T W}\left(\mathfrak{g}^{\Delta}\right), \operatorname{Tot}\left(\mathfrak{g}^{\Delta}\right), I, E$ and $h$ is functorial in the category DGLA $^{\Delta_{\text {mon }}}$ of semicosimplicial DGLAs.

In geometric applications, if $\mathcal{L} \rightarrow \mathcal{A}$ is an acyclic resolution, i.e., a quasi-isomorphism of sheaves of DGLAs such that $\mathcal{A}^{k}$ is an acyclic sheaf of $\mathcal{O}_{X}$-modules for every $k$, then one can use the augmentation $H^{0}(X ; \mathcal{A}) \rightarrow \mathcal{A}(\mathcal{U})$ to link the Thom-Whitney DGLA of $\mathcal{L}(\mathcal{U})$ with the DGLA of global sections of $\mathcal{A}$.

Theorem 3.7. Let $X$ be a paracompact Hausdorff topological space, $\mathcal{L}$ a sheaf of differential graded Lie algebras on $X$, and $\mathcal{L} \rightarrow \mathcal{A}$ an acyclic resolution. Then, if $\mathcal{U}$ is an open cover of $X$ which is acyclic with respect to both $\mathcal{L}$ and $\mathcal{A}$, the $D G L A \operatorname{Tot}_{T W}(\mathcal{L}(\mathcal{U}))$ is naturally quasi-isomorphic to the DGLA $H^{0}(X ; \mathcal{A})$.

Proof. We recall that an augmented semicosimplicial differential graded Lie algebra is a diagram $\mathfrak{g}_{-1} \rightarrow \mathfrak{g}^{\Delta}$, where $\mathfrak{g}^{\Delta}$ is a semicosimplicial DGLA and $\partial_{0,0}: \mathfrak{g}_{-1} \rightarrow \mathfrak{g}_{0}$ is a DGLA morphism such that $\partial_{0,1} \partial_{0,0}=\partial_{1,1} \partial_{0,0}$. There is a morphism of DGLAs

$$
\begin{aligned}
\mathfrak{g}_{-1} & \rightarrow \operatorname{Tot}_{T W}\left(\mathfrak{g}^{\Delta}\right), \\
x & \mapsto\left(\partial_{0,0} x, \partial_{1,1} \partial_{0,0} x, \partial_{2,2} \partial_{1,1} \partial_{0,0} x, \ldots\right),
\end{aligned}
$$

induced by the natural morphism $\lim \mathfrak{g}^{\Delta} \rightarrow \operatorname{holim} \mathfrak{g}^{\Delta}$. Since $\mathcal{A}$ is an acyclic resolution of $\mathcal{L}$, by a standard argument in homological algebra we have a chain of quasiisomorphisms of DGLAs

$$
H^{0}(X ; \mathcal{A}) \stackrel{\sim}{\rightarrow} \operatorname{Tot}_{T W}(\mathcal{A}(\mathcal{U})) \stackrel{\sim}{\leftarrow} \operatorname{Tot}_{T W}(\mathcal{L}(\mathcal{U}))
$$




\subsection{Truncations}

Let $\mathfrak{g}^{\Delta}$ be a semicosimplicial DGLA, and let $m_{1} \in \mathbb{N}$ and $m_{2} \in \mathbb{N} \cup\{\infty\}$ with $m_{1} \leq m_{2}$. We denote by $\mathfrak{g}^{\Delta_{\left[m_{1}, m_{2}\right]}}$ the semicosimplicial DGLA truncated between levels $m_{1}$ and $m_{2}$ defined by

$$
\left(\mathfrak{g}^{\left.\Delta_{\left[m_{1}, m_{2}\right]}\right)_{n}}= \begin{cases}\mathfrak{g}_{n} & \text { for } m_{1} \leq n \leq m_{2}, \\ 0 & \text { otherwise, }\end{cases}\right.
$$

with the obvious maps $\partial_{k, i}^{\left[m_{1}, m_{2}\right]}=\partial_{k, i}$ for $m_{1}<i \leq m_{2}$, and $\partial_{k, i}^{\left[m_{1}, m_{2}\right]}=0$, otherwise. For any nonnegative integers $m_{1}, m_{2}, r_{1}, r_{2}$ such that $r_{i} \leq m_{i}$, the map $\operatorname{Id}_{\left[m_{1}, r_{2}\right]}: \mathfrak{g}^{\Delta_{\left[m_{1}, m_{2}\right]}} \rightarrow$ $\mathfrak{g}^{\Delta_{\left[r_{1}, r_{2}\right]}}$ given by

$$
\left.\operatorname{Id}_{\left[m_{1}, r_{2}\right]}\right|_{\left(\mathfrak{g}^{\Delta\left[m_{1}, m_{2}\right]}\right)_{n}}= \begin{cases}\operatorname{Id}_{\mathfrak{g}_{n}} & \text { if } m_{1} \leq n \leq r_{2}, \\ 0 & \text { otherwise, }\end{cases}
$$

is a morphism of semicosimplicial DGLAs; it induces the natural morphism of complexes $\phi: \operatorname{Tot}\left(\mathfrak{g}^{\left.\Delta_{\left[m_{1}, m_{2}\right]}\right)} \rightarrow \operatorname{Tot}\left(\mathfrak{g}^{\Delta_{\left[r_{1}, r_{2}\right]}}\right)\right.$ and the natural morphism of DGLAs $\psi:$ $\operatorname{Tot}_{T W}\left(\mathfrak{g}^{\left.\Delta_{\left[m_{1}, m_{2}\right]}\right]}\right) \rightarrow \operatorname{Tot}_{T W}\left(\mathfrak{g}^{\Delta_{\left[r_{1}, r_{2}\right]}}\right)$. Note that we have a homotopy commutative diagram of complexes

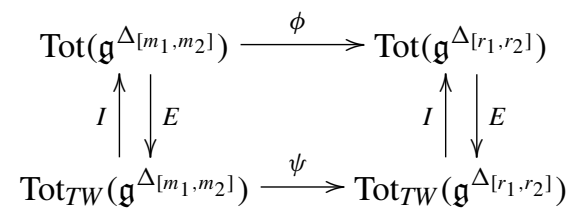

Proposition 3.8. Let $\mathfrak{g}^{\Delta}$ be a semicosimplicial DGLA such that $H^{j}\left(\mathfrak{g}_{i}\right)=0$ for all $i \geq 0$ and $j<0$. Then the morphism $\operatorname{Id}_{[0,2]}$ induces a natural isomorphism of functors

$$
\operatorname{Def}_{\operatorname{Tot}_{T W}\left(\mathfrak{g}^{\Delta}\right)} \stackrel{\sim}{\rightarrow} \operatorname{Def}_{\operatorname{Tot}_{T W}\left(\mathfrak{g}^{\Delta}[0,2]\right)} .
$$

Proof. It is a well known fact (see, e.g., [17] for a proof) that a DGLA morphism which is surjective on $H^{0}$, bijective on $H^{1}$ and injective on $H^{2}$ induces an isomorphism between the associated deformation functors. Since the above homotopy commutative diagram identifies $H^{*}(\psi)$ with $H^{*}(\phi)$, it is enough to prove that $H^{0}(\phi)$ is surjective, $H^{1}(\phi)$ is bijective and $H^{2}(\phi)$ is injective. This is easily checked by looking at the spectral sequences associated with double complexes of $\mathfrak{g}^{\Delta}$ and $\mathfrak{g}^{\Delta_{[0,2]}}$.

Remark 3.9. Observe that, for any semicosimplicial DGLA $\mathfrak{g}^{\Delta}$, we have $Z_{\mathrm{sc}}^{1}\left(\exp \mathfrak{g}^{\Delta}\right)=$ $Z_{\mathrm{sc}}^{1}\left(\exp \mathfrak{g}^{\Delta_{[0,2]}}\right)$ and $H_{\mathrm{sc}}^{1}\left(\exp \mathfrak{g}^{\Delta}\right)=H_{\mathrm{sc}}^{1}\left(\exp \mathfrak{g}^{\Delta_{[0,2]}}\right)$. Moreover, the inclusion $Z_{\mathrm{sc}}^{1}\left(\exp \mathfrak{g}^{\Delta}\right)$ $\hookrightarrow Z_{\mathrm{sc}}^{1}\left(\exp \mathfrak{g}^{\Delta_{[0,1]}}\right)$ induces an injective map $H_{\mathrm{sc}}^{1}\left(\exp \mathfrak{g}^{\Delta}\right) \hookrightarrow H_{\mathrm{sc}}^{1}\left(\exp \mathfrak{g}^{\Delta_{[0,1]}}\right)$.

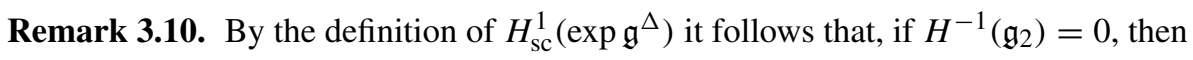

$$
T H_{\text {sc }}^{1}\left(\exp \mathfrak{g}^{\Delta}\right)=H^{1}\left(\operatorname{Tot}\left(\mathfrak{g}^{\Delta[0,2]}\right)\right) .
$$

Hence, the two functors of Artin rings $H_{\mathrm{sc}}^{1}\left(\exp \mathfrak{g}^{\Delta}\right)$ and $\operatorname{Def}_{\operatorname{Tot}_{T W}\left(\mathfrak{g}^{\Delta}[0,2]\right)}$ have naturally isomorphic tangent spaces when $H^{-1}\left(\mathfrak{g}_{2}\right)=0$. We will show in Section 4.3 that in this case these two functors are actually isomorphic. 


\section{An isomorphism of deformation functors}

\subsection{A lemma on Maurer-Cartan elements}

We will now give an explicit description of the solutions of the Maurer-Cartan equation for the DGLAs $\operatorname{Tot}_{T W}\left(\mathfrak{g}^{\Delta_{[0,1]}}\right)$ and $\operatorname{Tot}_{T W}\left(\mathfrak{g}^{\Delta_{[0,2]}}\right)$.

Proposition 4.1. Let $\mathfrak{g}^{\Delta}$ be a semicosimplicial DGLA. Then, for every $A \in \mathbf{A r t}_{\mathbb{K}}$, the solutions of the Maurer-Cartan equation for the Thom-Whitney DGLA $\operatorname{Tot}_{T W}\left(\mathfrak{g}^{\left.\Delta_{[0,1]}\right) \otimes}\right.$ $\mathfrak{m}_{A}$ are of the form $\left(x, e^{p(t)} * \partial_{0,1} x\right)$, where $x \in \operatorname{MC}_{\mathfrak{g}_{0}}(A)$ and $p(t) \in\left(\mathfrak{g}_{1}^{0}[t] \cdot t\right) \otimes \mathfrak{m}_{A}$. The elements $x, p$ are uniquely determined, and they satisfy

$$
\partial_{1,1} x=e^{p(1)} * \partial_{0,1} x
$$

The solutions of the Maurer-Cartan equation for the Thom-Whitney DGLA $\operatorname{Tot}_{T W}\left(\mathfrak{g}^{\Delta_{[0,2]}}\right)$ $\otimes \mathfrak{m}_{A}$ are of the form

$$
\left(x, e^{p(t)} * \partial_{0,1} x, e^{q\left(s_{0}, s_{1}\right)+r\left(s_{0}, s_{1}, d s_{0}, d s_{1}\right)} * \partial_{0,2} \partial_{0,1} x\right),
$$

where $x \in \mathrm{MC}_{\mathfrak{g}_{0}}(A), p(t) \in\left(\mathfrak{g}_{1}^{0}[t] \cdot t\right) \otimes \mathfrak{m}_{A}, q\left(s_{0}, s_{1}\right) \in\left(\mathfrak{g}_{2}^{0}\left[s_{0}, s_{1}\right] \cdot s_{0}+\mathfrak{g}_{2}^{0}\left[s_{0}, s_{1}\right] \cdot s_{1}\right)$ $\otimes \mathfrak{m}_{A}$ and $r\left(s_{0}, s_{1}, d s_{0}, d s_{1}\right) \in\left(\mathfrak{g}_{2}^{-1}\left[s_{0}, s_{1}\right] \cdot s_{0} d s_{1}\right) \otimes \mathfrak{m}_{A}$. The elements $x, p, q, r$ are uniquely determined, and they satisfy

$$
\left\{\begin{array}{l}
\partial_{1,1} x=e^{p(1)} * \partial_{0,1} x \\
\partial_{0,2} p(t)=q(0, t) \\
\partial_{1,2} p(t)=q(t, 0), \\
e^{\left(-\partial_{2,2} p(t)\right) \bullet(q(t, 1-t)+r(t, 1-t, d t)) \bullet(-q(0,1))} * \partial_{2,2} \partial_{0,1} x=\partial_{2,2} \partial_{0,1} x .
\end{array}\right.
$$

Proof. We recall [6, Proposition 7.2] that, if $(L, d,[]$,$) is a differential graded Lie$ algebra such that $L=M \oplus C \oplus D$ as graded vector spaces, with $M$ a differential graded subalgebra of $L$, and with $d: C \rightarrow D[1]$ an isomorphism of graded vector spaces, then, for every $A \in \mathbf{A r t}_{\mathbb{K}}$ there exists a bijection

$$
\alpha: \operatorname{MC}_{M}(A) \times\left(C^{0} \otimes \mathfrak{m}_{A}\right) \stackrel{\sim}{\rightarrow} \operatorname{MC}_{L}(A), \quad(x, c) \mapsto e^{c} * x
$$

To prove (4.1), notice that $\operatorname{Tot}_{T W}\left(\mathfrak{g}^{\Delta_{[0,1]}}\right)$ is a sub-DGLA of $\mathfrak{g}_{0} \oplus \Omega_{1} \otimes \mathfrak{g}_{1}$ and use the decomposition of $\Omega_{1} \otimes \mathfrak{g}_{1}$ given by

$$
M=\mathfrak{g}_{1}, \quad C=\mathfrak{g}_{1}[t] \cdot t, \quad D=d C .
$$

To prove (4.2), use the decomposition of $\Omega_{2} \otimes \mathfrak{g}_{2}$ given by

$$
M=\mathfrak{g}_{2}, \quad C=\mathfrak{g}_{2}\left[s_{0}, s_{1}\right] \cdot s_{0}+\mathfrak{g}_{2}\left[s_{0}, s_{1}\right] \cdot s_{1}+\mathfrak{g}_{2}\left[s_{0}, s_{1}\right] \cdot s_{0} d s_{1}, \quad D=d C .
$$


4.2. The isomorphism $\left.H_{\mathrm{sc}}^{1}\left(\exp ^{\Delta_{[0,1]}}\right) \cong \operatorname{Def}_{\operatorname{Tot}_{T W}\left(\mathfrak{g}^{\Delta}[0,1]\right.}\right)$

Proposition 4.2. Let $\mathfrak{g}^{\Delta}$ be a semicosimplicial DGLA. The map

$$
\Phi_{[0,1]}: \mathrm{MC}_{\operatorname{Tot}_{T W}\left(\mathfrak{g}^{\Delta}[0,1]\right)}(A) \rightarrow\left(\mathfrak{g}_{0}^{1} \oplus \mathfrak{g}_{1}^{0}\right) \otimes \mathfrak{m}_{A}
$$

given by $\left(x, e^{p(t)} * \partial_{0,1} x\right) \mapsto(x, p(1))$ induces a natural transformation of functors of Artin rings $\operatorname{Def}_{\operatorname{Tot}_{T W}\left(\mathfrak{g}^{\Delta}[0,1]\right)} \rightarrow H_{\mathrm{sc}}^{1}\left(\exp ^{\Delta_{[0,1]}}\right)$.

Proof. Clearly, if $\left(x, e^{p(t)} * \partial_{0,1} x\right) \in \mathrm{MC}_{\mathrm{Tot}_{T W}\left(\mathfrak{g}^{\Delta[0,1]}\right)}(A)$, then $(x, p(1)) \in Z_{\mathrm{sc}}^{1}\left(\exp ^{\Delta_{[0,1]}}\right)$. We have to show that if two elements $\eta_{0}=\left(x_{0}, e^{p_{0}(t)} * \partial_{0,1} x_{0}\right)$ and $\eta_{1}=\left(x_{1}, e^{p_{1}(t)} *\right.$ $\left.\partial_{0,1} x_{1}\right)$ in $\mathrm{MC}_{\operatorname{Tot}_{T W}\left(\mathfrak{g}^{\Delta}[0,1]\right)}(A)$ are homotopy equivalent, then $\Phi_{[0,1]}\left(\eta_{0}\right) \sim \Phi_{[0,1]}\left(\eta_{1}\right)$ in $Z_{\mathrm{sc}}^{1}\left(\exp \mathfrak{g}^{\Delta_{[0,1]}}\right)$. Let $z(\xi, d \xi)$ be a homotopy between $\eta_{0}$ and $\eta_{1}$. Therefore, $z(\xi, d \xi)$ is a Maurer-Cartan element for $\operatorname{Tot}_{T W}\left(\mathfrak{g}^{\Delta_{[0,1]}}\right)[\xi, d \xi]$ and so, reasoning as in the proof of Proposition 4.1, we find

$$
z(\xi, d \xi)=\left(e^{T(\xi)} * u, e^{U(t, d t ; \xi)} * v\right),
$$

with $T(0)=U(t, d t ; 0)=0$. Since $z(0)=\eta_{0}$, we get

$$
z(\xi, d \xi)=\left(e^{T(\xi)} * x_{0}, e^{U(t, d t ; \xi)} * e^{p_{0}(t)} * \partial_{0,1} x_{0}\right) .
$$

The face conditions for $z(\xi, d \xi)$ and uniqueness imply $U(0 ; \xi)=\partial_{0,1} T(\xi)$ and $U(1 ; \xi)$ $=\partial_{1,1} T(\xi)$. Moreover, $z(1)=\eta_{1}$, and so

$$
\left(e^{T(1)} * x_{0}, e^{U(t, d t ; 1)} * e^{p_{0}(t)} * \partial_{0,1} x_{0}\right)=\left(x_{1}, e^{p_{1}(t)} * \partial_{0,1} x_{1}\right)
$$

by uniqueness again, we have $e^{T(1)} * x_{0}=x_{1}$. Furthermore, $e^{U(t, d t ; 1)} * e^{p_{0}(t)} * \partial_{0,1} x_{0}=$ $e^{p_{1}(t)} * \partial_{0,1} x_{1}$, so, using the face conditions for $\eta_{0}$ and $\eta_{1}$, we obtain

$$
\partial_{0,1} x_{0}=e^{-p_{0}(t) \bullet-U(t, d t ; 1) \bullet p_{1}(t) \bullet \partial_{0,1} T(1)} * \partial_{0,1} x_{0} .
$$

Next, we recall [11, Lemma 6.15] that if $L$ is a DGLA and $x(t, d t)$ is a Maurer-Cartan element for $L[t, d t]$ and $\mu(t, d t) \in L[t, d t]^{0}$ is such that $e^{\mu(t, d t)} * x(t, d t)=x(t, d t)$, then $\mu(1)$ is an element of the irrelevant stabilizer of $x(1)$. Therefore, in our case we get

$$
-p_{0}(1) \bullet-\partial_{1,1} T(1) \bullet p_{1}(1) \bullet \partial_{0,1} T(1) \in \operatorname{Stab}\left(\partial_{0,1} x_{0}\right)
$$

Proposition 4.3. Let $\mathfrak{g}^{\Delta}$ be a semicosimplicial DGLA. The map

$$
\Phi_{[0,1]}: \operatorname{Def}_{\operatorname{Tot}_{T W}\left(\mathfrak{g}^{\Delta}[0,1]\right)} \rightarrow H_{\mathrm{sc}}^{1}\left(\exp \mathfrak{g}^{\Delta_{[0,1]}}\right)
$$

is an isomorphism of functors of Artin rings. In particular, $H_{\mathrm{sc}}^{1}\left(\exp ^{\Delta^{\Delta_{0,1]}}}\right)$ is a deformation functor. 
Proof. Let $\Psi_{[0,1]}: Z_{\mathrm{sc}}^{1}\left(\exp ^{\Delta_{[0,1]}}\right)(A) \rightarrow \operatorname{Tot}_{T W}\left(\mathfrak{g}^{\Delta_{[0,1]}}\right) \otimes \mathfrak{m}_{A}$ be the map given by $(l, m) \mapsto\left(l, e^{t m} * \partial_{0,1} l\right)$; it is immediate to check that $\Phi_{[0,1]}$ actually takes its values in $\mathrm{MC}_{\operatorname{Tot}_{T W}\left(\mathfrak{g}^{\Delta}[0,1]\right)}(A)$. Moreover, $\Psi_{[0,1]}$ induces a map

$$
H_{\mathrm{sc}}^{1}\left(\exp \mathfrak{g}^{\Delta_{[0,1]}}\right)(A) \rightarrow \operatorname{Def}_{\operatorname{Tot}_{T W}\left(\mathfrak{g}^{\Delta}[0,1]\right)}(A),
$$

which is the inverse of $\Phi_{[0,1]}$. Indeed, if $\left(l_{0}, m_{0}\right) \sim\left(l_{1}, m_{1}\right)$ in $Z_{\mathrm{sc}}^{1}\left(\exp \mathfrak{g}^{\Delta_{[0,1]}}\right)(A)$, then there exist elements $a \in \mathfrak{g}_{0}^{0} \otimes \mathfrak{m}_{A}$ and $b \in \mathfrak{g}_{1}^{-1} \otimes \mathfrak{m}_{A}$ such that

$$
\left\{\begin{array}{l}
e^{a} * l_{0}=l_{1} \\
-m_{0} \bullet-\partial_{1,1} a \bullet m_{1} \bullet \partial_{0,1} a=d b+\left[\partial_{0,1} l_{0}, b\right] .
\end{array}\right.
$$

Therefore, the images $\left(l_{0}, e^{t m_{0}} * \partial_{0,1} l_{0}\right)$ and $\left(l_{1}, e^{t m_{1}} * \partial_{0,1} l_{1}\right)$ are homotopic via the element

$$
z(\xi, d \xi)=\left(e^{\xi a} * l_{0}, e^{t\left(\partial_{1,1}(\xi a) \bullet m_{0} \bullet\left(d(\xi b)+\left[\partial_{0,1} l_{0}, \xi b\right]\right) \bullet-\partial_{0,1}(\xi a)\right) \bullet \partial_{0,1}(\xi a)} * \partial_{0,1} l_{0}\right) .
$$

The composition $\Phi_{[0,1]} \circ \Psi_{[0,1]}: Z_{\mathrm{sc}}^{1}\left(\exp ^{\Delta_{[0,1]}}\right)(A) \rightarrow Z_{\mathrm{sc}}^{1}\left(\exp ^{\Delta_{[0,1]}}\right)(A)$ is clearly the identity, whereas $\Psi_{[0,1]} \circ \Phi_{[0,1]}: \operatorname{MC}_{\operatorname{Tot}_{T W}\left(\mathfrak{g}^{\Delta}[0,1]\right)}(A) \rightarrow \operatorname{MC}_{\operatorname{Tot}_{T W}\left(\mathfrak{g}^{\Delta}[0,1]\right)}(A)$ is homotopic to the identity. Indeed, $\left(x, e^{p(t)} * \partial_{0,1} x\right)$ and $\left(x, e^{t p(1)} * \partial_{0,1} x\right)$ are homotopic in $\mathrm{MC}_{\operatorname{Tot}_{T W}\left(\mathfrak{g}^{\Delta}[0,1]\right)}(A)$ via the element $z(\xi, d \xi)=\left(x, e^{\xi t p(1)+(1-\xi) p(t)} * \partial_{0,1} x\right)$.

Remark 4.4. A particular case of Proposition 4.3, with an almost identical proof, has been considered by one of the authors in [11]. Namely, given three DGLAs $L, M$ and $N$ and two DGLA morphisms $h: L \rightarrow M$ and $g: N \rightarrow M$, one can consider the semicosimplicial DGLA

$$
L \oplus N \underset{(h, 0)}{\stackrel{(0, g)}{\rightrightarrows}} M \equiv 0 \Longrightarrow
$$

to recover [11, Theorem 6.17].

\subsection{Proof of the main theorem}

In this section, we prove the existence of a natural isomorphism of functors of Artin rings $H_{\text {sc }}^{1}\left(\exp \mathfrak{g}^{\Delta}\right) \cong \operatorname{Def}_{\operatorname{Tot}_{T W}\left(\mathfrak{g}^{\Delta}[0,2]\right)}$ for any semicosimplicial DGLA $\mathfrak{g}^{\Delta}$ such that $H^{-1}\left(\mathfrak{g}_{2}\right)$ $=0$. As an immediate consequence we obtain a natural isomorphism of deformation functors $H_{\mathrm{sc}}^{1}\left(\exp \mathfrak{g}^{\Delta}\right) \cong \operatorname{Def}_{\operatorname{Tot}_{T W}\left(\mathfrak{g}^{\Delta}\right)}$ for any semicosimplicial DGLA $\mathfrak{g}^{\Delta}$ such that $H^{j}\left(\mathfrak{g}_{i}\right)$ $=0$ for $i \geq 0$ and $j<0$.

The proof is considerably harder than in the case $\mathfrak{g}^{\Delta_{[0,1]}}$ considered in the previous section. Indeed, we are still able to define a map $\Phi: \mathrm{MC}_{\operatorname{Tot}_{T W}\left(\mathfrak{g}^{\Delta}[0,2]\right.} \rightarrow Z_{\mathrm{sc}}^{1}\left(\exp \mathfrak{g}^{\Delta}\right)$ inducing a natural transformation $\operatorname{Def}_{\operatorname{Tot}_{T W}\left(\mathfrak{g}^{\Delta}[0,2]\right)} \rightarrow H_{\mathrm{sc}}^{1}\left(\exp ^{\Delta}\right)$, but we will not be able to explicitly define a homotopy inverse to $\Phi$, so we will have to directly check that the map $\operatorname{Def}_{\operatorname{Tot}_{T W}\left(\mathfrak{g}^{\Delta}[0,2]\right)} \rightarrow H_{\mathrm{sc}}^{1}\left(\exp ^{\Delta}\right)$ is an isomorphism. 
Proposition 4.5. Let $\mathfrak{g}^{\Delta}$ be a semicosimplicial DGLA. The map

$$
\Phi: \mathrm{MC}_{\operatorname{Tot}_{T W}\left(\mathfrak{g}^{\Delta[0,2])}\right.}(A) \rightarrow\left(\mathfrak{g}_{0}^{1} \oplus \mathfrak{g}_{1}^{0}\right) \otimes \mathfrak{m}_{A}
$$

given by

$$
\left(x, e^{p(t)} * \partial_{0,1} x, e^{q\left(s_{0}, s_{1}\right)+r\left(s_{0}, s_{1}, d s_{1}, d s_{1}\right)} * \partial_{0,2} \partial_{0,1} x\right) \mapsto(x, p(1))
$$

induces a natural transformation of functors of Artin rings

$$
\operatorname{Def}_{\operatorname{Tot}_{T W}\left(\mathfrak{g}^{\Delta}[0,2]\right)} \rightarrow H_{\mathrm{sc}}^{1}\left(\exp \mathfrak{g}^{\Delta}\right)
$$

Proof. First we check that $\Phi$ takes its values in $Z_{\mathrm{sc}}^{1}\left(\exp ^{\Delta}\right)(A)$. The only nontrivial point consists in showing that $-\partial_{2,2} p(1) \bullet \partial_{1,2} p(1) \bullet-\partial_{0,2} p(1)$ is an element of the irrelevant stabilizer of $\partial_{2,2} \partial_{0,1} x$. This follows by the face condition

$$
e^{\left(-\partial_{2,2} p(t)\right) \bullet(q(t, 1-t)+r(t, 1-t, d t)) \bullet(-q(0,1))} * \partial_{2,2} \partial_{0,1} x=\partial_{2,2} \partial_{0,1} x,
$$

applying [11, Lemma 6.15] once again. Next, we notice that the equivalence relation on $Z_{\text {sc }}^{1}\left(\exp \mathfrak{g}^{\Delta}\right)(A)$ only involves the DGLAs $\mathfrak{g}_{0}$ and $\mathfrak{g}_{1}$; hence, we can conclude by following verbatim the proof of Proposition 4.2.

Proposition 4.6. The map $\Phi: \operatorname{Def}_{\operatorname{Tot}_{T W}\left(\mathfrak{g}^{\Delta[0,2]}\right)}(A) \rightarrow H_{\mathrm{sc}}^{1}\left(\exp \mathfrak{g}^{\Delta}\right)(A)$ is surjective for any $A \in \mathbf{A r t}_{\mathbb{K}}$.

Proof. Let $(l, m) \in Z_{\mathrm{sc}}^{1}\left(\exp ^{\Delta}\right)(A)$ and $n \in \mathfrak{g}_{2}^{-1} \otimes \mathfrak{m}_{A}$ be such that $\partial_{0,2} m \bullet-\partial_{1,2} m \bullet \partial_{2,2} m$ $=d n+\frac{1}{2}\left[\partial_{2,2} \partial_{0,1} l, n\right]$. Consider the element $w(t)=d(t n)+\frac{1}{2}\left[\partial_{2,2} \partial_{0,1} l, t n\right]$ in the irrelevant stabilizer of $\partial_{2,2} \partial_{0,1} l$ and

$$
R\left(s_{0}, s_{1}\right)=s_{0} s_{1} \frac{s_{0} \partial_{2,2} m \bullet-w\left(s_{0}\right) \bullet s_{0} \partial_{0,2} m \bullet-s_{0} \partial_{1,2} m}{s_{0}\left(1-s_{0}\right)} \bullet s_{0} \partial_{1,2} m \bullet s_{1} \partial_{0,2} m .
$$

Then

$$
\left(l, e^{t m} * \partial_{0,1} l, e^{R\left(s_{0}, s_{1}\right)} * \partial_{0,2} \partial_{0,1} l\right)
$$

is an element in $\mathrm{MC}_{\operatorname{Tot}_{T W}\left(\mathfrak{g}^{\Delta}{ }^{[0,2]}\right)}(A)$ in the fiber of $\Phi$ over $(l, m)$.

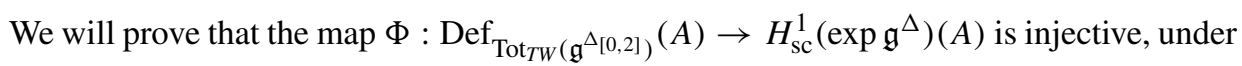
the hypothesis $H^{-1}\left(\mathfrak{g}_{2}\right)=0$. For this we need two remarks.

Remark 4.7. Let $(L, d,[]$,$) be a DGLA, A \in \operatorname{Art}_{\mathbb{K}}$ and $x \in L^{1} \otimes \mathfrak{m}_{A}$. The linear endomorphism $d_{x}=d+[x$,$] of L \otimes \mathfrak{m}_{A}$ is a differential if and only if $x \in \operatorname{MC}_{L}(A)$, and in this case $\left(L \otimes \mathfrak{m}_{A}, d_{x},[],\right)$ is a DGLA. So, we can define the set of MaurerCartan elements $\mathrm{MC}_{L}^{x}(A)$ and the gauge action of $\left(L^{0} \otimes \mathfrak{m}_{A}, d_{x},[],\right)$ on it. We denote by $\operatorname{Def}_{L}^{x}(A)$ the quotient of $\operatorname{MC}_{L}^{x}(A)$ with respect to the gauge action. The affine map

$$
L \otimes \mathfrak{m}_{A} \rightarrow L \otimes \mathfrak{m}_{A}, \quad v \mapsto v-x,
$$

induces an isomorphism $\operatorname{Def}_{L}(A) \cong \operatorname{Def}_{L}^{x}(A)$ with obvious inverse $v \mapsto v+x$. 
Next, let $M \subseteq L$ be a sub-DGLA and let $x \in \operatorname{MC}_{L}(A)$. If $M \otimes \mathfrak{m}_{A}$ is closed under the differential $d_{x}$, then we can consider the set of Maurer-Cartan elements $\operatorname{MC}_{M}^{x}(A)$, and its quotient $\operatorname{Def}_{M}^{x}(A)$. The tangent space to $\operatorname{Def}_{M}^{x}(A)$ is $H^{1}\left(M \otimes \mathfrak{m}_{A}, d_{x}\right)$; so, by upper semicontinuity of cohomology, $H^{1}(M, d)=0$ implies that $\operatorname{Def}_{M}^{x}(A)$ is trivial for all $x \in \mathrm{MC}_{L}(A)$ such that $d_{x}\left(M \otimes \mathfrak{m}_{A}\right) \subseteq M \otimes \mathfrak{m}_{A}$.

Remark 4.8. For any semicosimplicial DGLA $\mathfrak{g}^{\Delta}$, the truncation morphism

$$
\operatorname{Tot}_{T W}^{0}\left(\mathfrak{g}^{\Delta_{[0,2]}}\right) \rightarrow \operatorname{Tot}_{T W}^{0}\left(\mathfrak{g}^{\Delta_{[0,1]}}\right)
$$

is surjective, i.e., for any $\left(a_{0}, a_{1}\right) \in \operatorname{Tot}_{T W}^{0}\left(\mathfrak{g}^{\Delta_{[0,1]}}\right)$ there exists $a_{2} \in\left(\mathfrak{g}_{2} \otimes \Omega_{2}\right)^{0}$ such that $\left(a_{0}, a_{1}, a_{2}\right) \in \operatorname{Tot}_{T W}^{0}\left(\mathfrak{g}^{\Delta_{[0,2]}}\right)$.

Proposition 4.9. Let $\mathfrak{g}^{\Delta}$ be a semicosimplicial DGLA such that $H^{-1}\left(\mathfrak{g}_{2}\right)=0$. Then the map $\Phi: \operatorname{Def}_{\operatorname{Tot}_{T W}\left(\mathfrak{g}^{\Delta}[0,2]\right)}(A) \rightarrow H_{\mathrm{sc}}^{1}\left(\exp \mathfrak{g}^{\Delta}\right)(A)$ is injective for any $A \in \mathbf{A r t}_{K}$.

Proof. Consider the commutative diagram

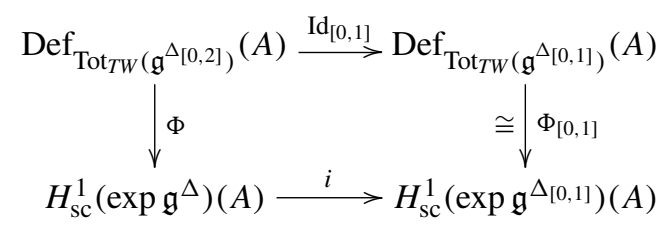

Since the map $\Phi_{[0,1]}$ is an isomorphism by Proposition 4.3, it is sufficient to prove that $\operatorname{Id}_{[0,1]}$ is injective. Let $\left(x_{0}, x_{1}, x_{2}\right)$ and $\left(x_{0}^{\prime}, x_{1}^{\prime}, x_{2}^{\prime}\right)$ be two Maurer-Cartan elements for $\operatorname{Tot}_{T W}\left(\mathfrak{g}^{\Delta_{[0,2]}}\right)$ such that $\left(x_{0}, x_{1}\right)$ and $\left(x_{0}^{\prime}, x_{1}^{\prime}\right)$ are gauge equivalent Maurer-Cartan elements for $\operatorname{Tot}_{T W}\left(\mathfrak{g}^{\Delta_{[0,1]}}\right)$. Let $\left(a_{0}, a_{1}\right) \in \operatorname{Tot}_{T W}^{0}\left(\mathfrak{g}^{\Delta_{[0,1]}}\right) \otimes \mathfrak{m}_{A}$ be an element realizing the gauge equivalence between $\left(x_{0}^{\prime}, x_{1}^{\prime}\right)$ and $\left(x_{0}, x_{1}\right)$, and let $\left(a_{0}, a_{1}, a_{2}\right)$ be a lift of $\left(a_{0}, a_{1}\right)$ in $\operatorname{Tot}_{T W}^{0}\left(\mathfrak{g}^{\Delta_{[0,2]}}\right) \otimes \mathfrak{m}_{A}$ (see Remark 4.8). Then $\left(x_{0}^{\prime}, x_{1}^{\prime}, x_{2}^{\prime}\right)$ is gauge equivalent via $\left(a_{0}, a_{1}, a_{2}\right)$ to the Maurer-Cartan element $\left(x_{0}, x_{1}, e^{a_{2}} * x_{2}^{\prime}\right)$ and it remains to prove that $\left(x_{0}, x_{1}, e^{a_{2}} * x_{2}^{\prime}\right)$ is gauge equivalent to $\left(x_{0}, x_{1}, x_{2}\right)$.

To see this, consider the DGLA $\operatorname{Tot}_{T W}\left(\mathfrak{g}^{\Delta_{[0,2]}}\right) \otimes \mathfrak{m}_{A}$ and modify its differential with the Maurer-Cartan element $\left(x_{0}, x_{1}, x_{2}\right)$, as in Remark 4.7. Translation by $\left(x_{0}, x_{1}, x_{2}\right)$ gives an isomorphism

$$
\operatorname{Def}_{\operatorname{Tot}_{T W}\left(\mathfrak{g}^{\Delta}[0,2]\right)}(A) \cong \operatorname{Def}_{\operatorname{Tot}_{T W}\left(\mathfrak{g}^{\Delta[0,2]}\right)}^{\left(x_{0}, x_{1}, x_{2}\right)}(A) ;
$$

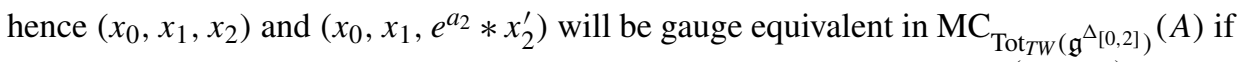
and only if $(0,0,0)$ and $\left(0,0, e^{a_{2}} * x_{2}^{\prime}-x_{2}\right)$ are gauge equivalent in $\operatorname{MC}_{\operatorname{Tot}_{T W}^{\left(x_{0}, x_{1}, x_{2}\right)}}^{(A, 2])}(A)$. Next, observe that the sub-DGLA Tot $T W\left(\mathfrak{g}^{\Delta_{[2,2]}}\right) \otimes \mathfrak{m}_{A}$ of $\operatorname{Tot}_{T W}\left(\mathfrak{g}^{\Delta_{[0,2]}}\right) \otimes \mathfrak{m}_{A}$ is closed under the modified differential $d_{\left(x_{0}, x_{1}, x_{2}\right)}$, so we can consider the deformation functor $\operatorname{Def}_{\operatorname{Tot}_{T W}\left(\mathfrak{g}^{\Delta_{[2,2]}}\right)}^{\left(x_{0}, x_{1}, x_{2}\right)}(A)$. Since $H^{1}\left(\operatorname{Tot}_{T W}\left(\mathfrak{g}^{\Delta_{[2,2]}}\right)\right)=H^{1}\left(\operatorname{Tot}\left(\mathfrak{g}^{\Delta_{[2,2]}}\right)\right)=H^{-1}\left(\mathfrak{g}_{2}\right)=0$, this deformation functor is trivial (see Remark 4.7). Therefore $\left(0,0, e^{a_{2}} * x_{2}^{\prime}-x_{2}\right)$ is gauge 
equivalent to $(0,0,0)$ as an element of $\mathrm{MC}_{\operatorname{Tot}_{T W}\left(\mathfrak{g}^{\Delta}[2,2]\right)}^{\left(x_{0}, x_{1}, x_{2}\right)}(A)$, and so, a fortiori, as an element of $\mathrm{MC}_{\operatorname{Tot}_{T W}\left(\mathfrak{g}^{\Delta}[0,2]\right)}^{\left(x_{0}, x_{1}, x_{2}\right)}(A)$.

Summing up, and recalling Proposition 3.8, we have proved:

Theorem 4.10. Let $\mathfrak{g}^{\Delta}$ be a semicosimplicial DGLA with $H^{-1}\left(\mathfrak{g}_{2}\right)=0$. Then there is a natural isomorphism of functors $\operatorname{Def}_{\operatorname{Tot}_{T W}\left(\mathfrak{g}^{\Delta}[0,2]\right)} \cong H_{\mathrm{sc}}^{1}\left(\exp \mathfrak{g}^{\Delta}\right)$. If moreover $H^{j}\left(\mathfrak{g}_{i}\right)=0$ for all $i \geq 0$ and $j<0$, then there is a natural isomorphism of functors $\operatorname{Def}_{\operatorname{Tot}_{T W}\left(\mathfrak{g}^{\Delta}\right)} \cong$ $H_{\mathrm{sc}}^{1}\left(\exp \mathfrak{g}^{\Delta}\right)$. In particular, in this case, the tangent space to $H_{\mathrm{sc}}^{1}\left(\exp \mathfrak{g}^{\Delta}\right)$ is $H^{1}\left(\operatorname{Tot}\left(\mathfrak{g}^{\Delta}\right)\right)$ and obstructions are contained in $H^{2}\left(\operatorname{Tot}\left(\mathfrak{g}^{\Delta}\right)\right)$.

As an immediate geometric application, we obtain:

Theorem 4.11. Let $X$ be a paracompact Hausdorff topological space, and let $\mathcal{L}$ be a sheaf of differential graded Lie algebras on $X$ such that the DGLAs $\mathcal{L}\left(U_{i_{0} \ldots i_{k}}\right)$ have no negative cohomology. Then every refinement $\mathcal{U}^{\prime} \geq \mathcal{U}$ of open covers of $X$ induces a nat-

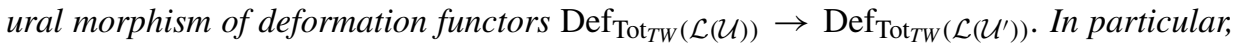
the direct limit

$$
\operatorname{Def}_{[\mathcal{L}]}=\underset{\overrightarrow{\mathcal{U}}}{\lim } \operatorname{Def}_{\operatorname{Tot}_{T W}(\mathcal{L}(\mathcal{U}))}
$$

is well defined and there is a natural isomorphism of functors of Artin rings

$$
H_{\mathrm{Ho}}^{1}(X ; \exp \mathcal{L}) \cong \operatorname{Def}_{[\mathcal{L}]} .
$$

Moreover, if acyclic open covers for $\mathcal{L}$ are cofinal in the directed family of all open covers of $X$, then

$$
H_{\mathrm{Ho}}^{1}(X ; \exp \mathcal{L}) \cong H_{\mathrm{sc}}^{1}(\exp \mathcal{L}(\mathcal{U})) \quad \text { and } \quad \operatorname{Def}_{[\mathcal{L}]} \cong \operatorname{Def}_{\operatorname{Tot}_{T W}(\mathcal{L}(\mathcal{U}))}
$$

for every $\mathcal{L}$-acyclic open cover $\mathcal{U}$ of $X$.

Proof. Let $\mathcal{U}^{\prime} \geq \mathcal{U}$ be a refinement of open covers of $X$, and let $\tau$ be a refinement function; it induces a natural morphism of semicosimplicial Lie algebras $\mathcal{L}(\mathcal{U}) \rightarrow \mathcal{L}\left(\mathcal{U}^{\prime}\right)$ and so a commutative diagram of natural transformations

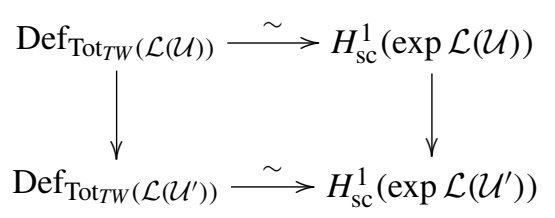

Horizontal arrows are isomorphisms by Theorem 4.10, and the right vertical arrow is independent of the refinement function $\tau$, as observed at the end of Section 3.1. Hence, the left morphism is also independent of $\tau$, so the direct limit

$$
\operatorname{Def}_{[\mathcal{L}]}=\underset{\mathcal{U}}{\lim } \operatorname{Def}_{\operatorname{Tot}_{T W}(\mathcal{L}(\mathcal{U}))}
$$


is well defined and we have a natural isomorphism $\operatorname{Def}_{[\mathcal{L}]} \cong H_{\mathrm{Ho}}^{1}(X ; \exp \mathcal{L})$. Assume now that acyclic open covers for $\mathcal{L}$ are cofinal in the family of all open covers of $X$. Then, for any refinement $\mathcal{U}^{\prime} \geq \mathcal{U}$ of acyclic open covers, the DGLA-morphism $\operatorname{Tot}_{T W}(\mathcal{L}(\mathcal{U})) \rightarrow$ $\operatorname{Tot}_{T W}\left(\mathcal{L}\left(\mathcal{U}^{\prime}\right)\right)$ is a quasi-isomorphism by Leray's theorem. Therefore, the left vertical arrow in the above diagram is an isomorphism and hence so is the right vertical one. Taking the direct limit over $\mathcal{L}$-acyclic covers, we conclude that, if $\mathcal{U}$ is an $\mathcal{L}$-acyclic open

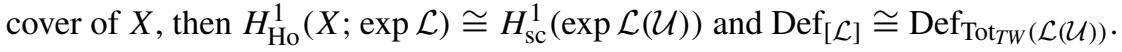

\section{Conclusions and further developments}

We can now sum up our results to obtain a DGLA description of infinitesimal deformations of a coherent sheaf. In Section 2, we analysed infinitesimal deformations of a coherent sheaf $\mathcal{F}$ of $\mathcal{O}_{X}$-modules on a ringed space $\left(X, \mathcal{O}_{X}\right)$. If $\mathcal{E} \rightarrow \mathcal{F} \rightarrow 0$ is a locally free resolution of $\mathcal{F}$ on $X$, we showed how infinitesimal deformations of $\mathcal{F}$ can be expressed in terms of the sheaf of DGLAs $\mathcal{E} n d^{*}(\mathcal{E})$. More precisely, in Section 3.1, we showed that the functor of infinitesimal deformations of $\mathcal{F}$ is isomorphic to $H_{\mathrm{Ho}}^{1}\left(X ; \exp \mathcal{E n d}^{*}\left(\mathcal{E}^{*}\right)\right)$.

Since negative Ext-groups between coherent sheaves are always trivial, all terms in the semicosimplicial DGLA $\mathcal{E} n d^{*}(\mathcal{E})(\mathcal{U})$ have zero negative cohomology. Therefore, Theorem 4.10 applies and we deduce that the functor of infinitesimal deformations of $\mathcal{F}$ is isomorphic to $\operatorname{Def}_{\left[\mathcal{E} n d^{*}(\mathcal{E})\right]}$; in particular, we recover the well known fact that the tangent space to $\operatorname{Def}_{\mathcal{F}}$ is $\operatorname{Ext}^{1}(\mathcal{F}, \mathcal{F})$ and that its obstructions are contained in $\operatorname{Ext}^{2}(\mathcal{F}, \mathcal{F})$.

Moreover, if $X$ is a smooth complex variety, then the DGLA controlling infinitesimal deformations of $\mathcal{F}$ turns out to be not at all mysterious. Indeed, let $\mathcal{E} n d^{*}(\mathcal{E}) \rightarrow$ $\mathcal{A}_{X}^{0, *}\left(\mathcal{E} n d^{*}\left(\mathcal{E}^{*}\right)\right)$ be the Dolbeault resolution of $\mathcal{E} n d^{*}\left(\mathcal{E}^{*}\right)$. Since this resolution is fine, by Theorem 3.7 the functor of infinitesimal deformations of $\mathcal{F}$ is isomorphic to the deformation functor associated with the DGLA $A_{X}^{0, *}\left(\mathcal{E} n d^{*}\left(\mathcal{E}^{*}\right)\right)$ of global sections of $\mathcal{A}_{X}^{0, *}\left(\mathcal{E} n d^{*}\left(\mathcal{E}^{*}\right)\right)$. We can also give an explicit description of this isomorphism of deformation functors. Indeed, a natural isomorphism

$$
\operatorname{Def}_{A_{X}^{0, *}\left(\mathcal{E} n d^{*}(\mathcal{E} \cdot)\right)}(B) \rightarrow \operatorname{Def}_{\mathcal{F}}(B) \quad \text { for } B \in \operatorname{Art}_{\mathbb{K}}
$$

is defined by associating with every Maurer-Cartan element $\xi$ of the DGLA $A_{X}^{0, *}\left(\mathcal{E} n d^{*}\left(\mathcal{E}^{*}\right)\right)$ the cohomology sheaf of $\left(\mathcal{A}_{X}^{0, *}(\mathcal{E}) \otimes B, \bar{\partial}+d_{\mathcal{E}}+\xi\right)$. Note that, by semicontinuity, this cohomology sheaf is concentrated in degree zero.

The techniques developed in this paper apply to a wide range of other geometric examples. More explicitly, we can use them in all cases when local deformations admit a simple DGLA description in terms of a resolution of the object to be deformed, for instance, in the case of infinitesimal deformations of a singular variety. Namely, let $X$ be a singular variety, $\mathcal{O}_{X}$ the sheaf of regular function of $X$, and $\mathcal{R} \rightarrow \mathcal{O}_{X}$ its standard free resolution [12, Section 1.5]. Then the deformation functor of infinitesimal deformations of $X$ is isomorphic to $H_{\mathrm{Ho}}^{1}\left(X\right.$; $\left.\exp \operatorname{Der}^{*}\left(\mathcal{R}^{*}\right)\right)$; see [5] for details. From this, we also recover the classical result that the tangent space to deformations of $X$ is $\operatorname{Ext}^{1}\left(\mathbb{L}_{X}, \mathcal{O}_{X}\right)$, 
and that obstructions are contained in $\operatorname{Ext}^{2}\left(\mathbb{L}_{X}, \mathcal{O}_{X}\right)$, where $\mathbb{L}_{X}$ is the cotangent complex of $X$.

Acknowledgments. We thank the referee for careful reading of a preliminary draft of this paper. We thank Marco Manetti for stimulating discussions on the subject and for useful comments and suggestions; D.I. thanks the Mathematical Department "Guido Castelnuovo", Sapienza Università di Roma, for its hospitality.

\section{References}

[1] Artin, M.: Deformation of singularities. Tata Institute of Fundamental Research, Bombay (1976)

[2] Cheng, X. Z., Getzler, E.: Transferring homotopy commutative algebraic structures. J. Pure Appl. Algebra 212, 2535-2542 (2008) Zbl 1158.18006 MR 2440265

[3] Dupont, J. L.: Simplicial de Rham cohomology and characteristic classes of flat bundles. Topology 15, 233-245 (1976) Zbl 0331.55012 MR 0413122

[4] Dupont, J. L.: Curvature and Characteristic Classes. Lecture Notes in Math. 640, Springer (1978) Zbl 0373.57009 MR 0500997

[5] Fiorenza, D., Iacono, D., Martinengo, E.: Infinitesimal deformations of singular varieties (in preparation)

[6] Fiorenza, D., Manetti, M.: $L_{\infty}$-structures on mapping cones. Algebra Number Theory 1, 301330 (2007) Zbl 1166.17010 MR 2361936

[7] Fiorenza, D., Manetti, M., Martinengo, E.: Semicosimplicial DGLAs in deformation theory. arXiv:math.AG/08030399

[8] Getzler, E.: Lie theory for nilpotent $L_{\infty}$-algebras. Ann. of Math. 170, 271-301 (2009) Zbl pre05578961 MR 2521116

[9] Hinich, V.: Descent of Deligne groupoids. Int. Math. Res. Notices 1997, no. 5, 223-239 Zbl 0948.22016 MR 1439623

[10] Hirschowitz, A., Simpson, C.: Descent pour les $n$-champs. arXiv:9807049v3

[11] Iacono, D.: $L_{\infty}$-algebras and deformations of holomorphic maps. Int. Math. Res. Notices 2008, no. 8, art. ID rnn013, 36 pp. Zbl pre05306018 MR 2428147

[12] Illusie, L.: Complexe cotangent et déformations I, II. Lecture Notes in Math. 239, 283, Springer, New York/Berlin (1971, 1972) Zbl 0224.13014(I) Zbl 0238.13017(II) MR 0491680(I) MR 0491681(II)

[13] Kodaira, K.: Complex Manifolds and Deformation of Complex Structures. Grundlehren Math. Wiss. 283, Springer (1986) Zbl 0581.32012 MR 0815922

[14] Kodaira, K., Spencer, D. C.: On deformations of complex analytic structures, II. Ann. of Math. 67, 403-466 (1958) Zbl 0128.16901

[15] Kuranishi, M.: Deformations of Compact Complex Manifolds. Sém. Math. Sup. 39, Presses Univ. de Montréal, Montreal (1971) Zbl 0382.32014

[16] Lurie, J.: Higher Topos Theory. Ann. of Math. Stud. 170, Princeton Univ. Press, Princeton, NJ (2009) Zbl 1175.18001 MR 2522659

[17] Manetti, M.: Deformation Theory via Differential Graded Lie Algebras. Seminari di Geometria Algebrica 1998-1999, Scuola Normale Superiore (1999) MR 1754793

[18] Manetti, M.: Extended deformation functors. Int. Math. Res. Notices 2002, no. 14, 719-756 Zbl 1063.58007 MR 1891232

[19] Navarro Aznar, V.: Sur la théorie de Hodge-Deligne. Invent. Math. 90, 11-76 (1987) Zbl 0639.14002 MR 0906579 
[20] Pridham, J. P.: Deformations via simplicial deformation complexes. arXiv:math/0311168v6

[21] Quillen, D.: Rational homotopy theory. Ann. of Math. (2) 90, 205-295 (1969) Zbl 0191.53702 MR 0258031

[22] Seidel, P., Thomas, R. P.: Braid group actions on derived categories of coherent sheaves. Duke Math. J. 108, 37-108 (2001) Zbl 1092.14025 MR 1831820

[23] Sernesi, E.: Deformations of Algebraic Schemes. Grundlehren Math. Wiss. 334, Springer (2006) Zbl 1102.14001 MR 2247603

[24] Toën, B.: Higher and derived stack: a global overview. In: Algebraic Geometry-Seattle 2005, Proc. Sympos. Pure Math. 80, Part 1, Amer. Math. Soc., Providence, RI, 435-487 (2009) Zbl 1183.14001 MR 2483943

[25] Yekutieli, A.: Twisted deformation quantization of algebraic varieties. arXiv:0905.0488v2 